\title{
LncRNA BC200/miR-150-5p/MYB positive feedback loop promotes the malignant proliferation of myelodysplastic syndrome
}

\author{
Zhaoping Liu ${ }^{1,2,7}$, Pan Wang ${ }^{3,7}$, Shunling Yuan ${ }^{1,2,7}$, Yanyan Wang ${ }^{2}$, Pengfei Cao ${ }^{4}$, Feng Wen ${ }^{5}$, Hui Li (iD ${ }^{3}$, Lin Zhu ${ }^{3}$, Long Liang ${ }^{3}$, \\ Zi Wang $\mathbb{D}^{3}$, Bin $\mathrm{Hu}^{3}$, Fuxiang Zheng ${ }^{1}$, Jing Liu $\mathbb{D}^{3,6 凶}$, Xiaojuan Xiao $\mathbb{D}^{3 凶}$ and Ji Zhang $\mathbb{D}^{1,2 \bowtie}$
}

(c) The Author(s) 2022

\begin{abstract}
Myelodysplastic syndrome (MDS) is a group of heterogeneous hematologic malignancies with a risk of transformation to acute myeloid leukemia. Understanding the molecular mechanisms of the specific roles of long noncoding RNAs (IncRNAs) in MDS would create novel ways to identify diagnostic and therapeutic targets. The IncRNA BC200 is upregulated and acts as an oncogene in various cancers; however, its expression, clinical significance, and roles in MDS remain unclear. Here, we found that BC200 was highly expressed in MDS patients compared with normal individuals. Knockdown of BC200 inhibited MDS cell proliferation, colony formation, and cell cycle progression in vitro and suppressed the growth and invasiveness of MDS cells in vivo. Mechanistic investigations revealed that BC200 functioned as a miRNA sponge to positively regulate the expression of MYB through sponging miR-150-5p and subsequently promoted malignant proliferation of MDS cells. Conversely, we found that BC200 was a direct transcriptional target of MYB, and knockdown of MYB abolished the oncogenic effect of BC200/miR-150-5p. Taken together, our results revealed that the BC200/miR-150-5p/MYB positive feedback loop promoted the proliferation of MDS cells and is expected to be a potential biomarker and therapeutic target in MDS.
\end{abstract}

Cell Death and Disease (2022)13:126; https://doi.org/10.1038/s41419-022-04578-2

\section{INTRODUCTION}

Myelodysplastic syndrome (MDS) is a clonal hematological malignancy characterized by ineffective hematopoiesis, progressive cytopenia and clonal evolution to acute myeloid leukemia $[1,2]$. The pathophysiology of MDS is a multistep process involving cytogenetic changes, gene mutations, or both. MDS frequently has unbalanced translocations and deletions, which implicates the loss of tumor suppressor gene function or the activation of oncogenes [3]. Malignant clonal hematopoietic cells often coexist and compete with normal hematopoietic cells for a considerable period of time in the bone marrow (BM) of MDS patients. When malignant clonal cells become dominant in $B M$, the disease progresses to AML [4]. The current treatment approaches include hypomethylation chemotherapy and immunomodulatory drugs. Patients with MDS are usually at an advanced age and may have comorbidities that make them ineligible for these treatment options due to excessive toxicity [5]. Thus, better understanding of the pathogenesis of MDS is crucial for developing novel and effective treatment strategies against this fatal disease.
Increasing evidence has shown that long noncoding RNAs (IncRNAs) play crucial roles in contributing to the pathogenesis and progression of various tumors $[6,7]$. LncRNAs are a class of transcripts that are $>200$ nucleotides and do not contain any extended open reading frames [8]. LncRNAs participate in the regulation of target gene expression through various mechanisms, and the most common regulatory approach for IncRNA regulating its target gene is to act as microRNA (miRNA) sponges [9]. In the IncRNA-miRNA-mRNA regulatory network, IncRNA acts as competing endogenous RNAs (ceRNAs) of specific mRNAs [10]. Numerous studies have shown that IncRNAs are frequently dysregulated in various cancers and are involved in a wide range of biological processes, such as malignant proliferation, migration, invasion, and metastasis [11, 12]. The aberrant expression signatures of IncRNAs have been revealed, which provides new insights into the biology of MDS. For instance, IncRNA HOXB-AS3 was found to be upregulated in MDS and was associated with poor prognosis [13]. Moreover, deregulation of several IncRNAs, such as H19, LEF1-AS1WT1-AS, and TCL6, is associated with higher-risk MDS [14]. However, the biological mechanisms of numerous IncRNAs in the pathogenesis and progression of MDS need to be further explored.

\footnotetext{
${ }^{1}$ Department of Clinical Laboratory, Shenzhen Traditional Chinese Medicine Hospital, Shenzhen, 518033 Guangdong, China. ${ }^{2}$ The First Affiliated Hospital, Department of Clinical Laboratory, Hengyang Medical School, University of South China, Hengyang 421001 Hunan, China. ${ }^{3}$ Molecular Biology Research Center \& Center for Medical Genetics, School of Life Sciences, Central South University, Changsha 410078 Hunan, China. ${ }^{4}$ Department of Hematology, Xiangya Hospital, Central South University, Changsha 410008, China. ${ }^{5}$ The First Affiliated Hospital, Department of Hematology, Hengyang Medical School, University of South China, Hengyang 421001 Hunan, China.

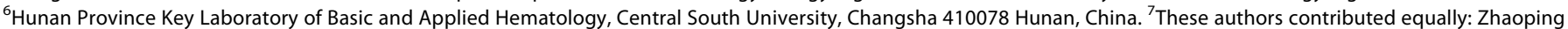
Liu, Pan Wang, Shunling Yuan. ${ }^{凶}$ email: liujing2@sklmg.edu.cn; xiaojuan_xiao@csu.edu.cn; zhang_ji001@hotmail.com Edited by Professor Massimiliano Agostini
} 
Recently, a growing number of studies have revealed that BC200, also known as brain cytoplasmic RNA 1 (BCYRN1), is elevated in a variety of cancer cells, including lung, breast, colorectal, gastric, ovary, and cervix [15-18]. Oncogenic BC200 plays an important role in the proliferation, apoptosis, migration, and invasion of cancer cells [15, 16, 19]. Furthermore, the expression of $\mathrm{BC} 200$ is regulated by several factors, including estrogen receptor- $a$ and c-myc. Estrogen induced BC200 expression, which promotes the growth and tumorsphere formation of hepatocellular carcinoma [20]. In non-small-cell lung cancer, BC200 is activated by the transcription factor c-myc, which is critical for cell migration and invasion [21]. However, how BC200 is transcriptionally regulated in MDS and whether it is involved in the pathogenesis and development of MDS are still unclear.

In this study, oncogenic BC200 promoted the growth of MDS cells in vitro and in vivo. Mechanistically, BC200 acted as a sponge for miR$150-5 p$ to upregulate the expression and activity of MYB. Interestingly, MYB, a crucial transcription factor, could directly bind to the BC200 promoter region and induce $\mathrm{BC} 200$ transcription, which in turn increased MYB expression. Furthermore, the high expression of BC200 and MYB was significantly negatively correlated with miR-150-5p in MDS patients and MDS cell lines. Thus, our results indicated that the BC200/miR-150-5p/MYB positive feedback loop contributed to the malignant proliferation of MDS cells, providing novel insight into the growth mechanism of MDS and identifying promising therapeutic targets for MDS treatment.

\section{MATERIALS AND METHODS \\ Cell lines and MDS samples}

Human MDS cell lines (SKM-1 and MDS-L) and HEK293T cell lines were purchased from ATCC (Manassas, VA, USA). SKM-1 cells were maintained in RPMI-1640 (Gibco, Rockville, MD, USA). MDS-L cells were cultured in RPMI1640 medium plus $50 \mu \mathrm{M}$ 2-mercaptoethanol and $100 \mathrm{U} / \mathrm{ml}$ IL-3. HEK293T cells were cultured in Dulbecco's modified Eagle's medium (Gibco). All media were supplemented with $10 \%$ fetal bovine serum (Gibco) and $1 \%$ penicillin-streptomycin (Gibco). All cells were cultured at $37^{\circ} \mathrm{C}$ in a $5 \% \mathrm{CO}_{2}$ atmosphere. The cells were authenticated using STR profiles. All cells were routinely tested as mycoplasma-free. All clinical samples were obtained with informed consent at the First Affiliated Hospital of University of South China (Hengyang, China) and Xiangya Hospital of Central South University (Changsha, China). Sample collection was approved by the Hospital's Protection of Human Subjects Committee. MDS primary samples were cultured in RPMI- 1640 medium containing $20 \%$ fetal bovine serum and $100 \mathrm{U} / \mathrm{ml} \mathrm{IL-3.}$

\section{Plasmids and transfection}

The miR-150-5p inhibitor and inhibitor control, miR-150-5p mimics and miRNA mimics control, small interfering RNAs (siRNAs) targeting BC200 and MYB, and an unrelated sequence used as a negative control siRNA were constructed by RiboBio (Guangzhou, China). The sequences of the reagents were listed in Supplementary Table S1. The CDNA expression plasmids pCMV3-BC200, pCMV3-MYB, and pCMV3-control vector were purchased from Sino Biological (Beijing, China). For the generation of stable cell lines, BC200-specific short hairpin RNA (shRNA) or control vector (GenePharma, Shanghai, China) were transfected into MDS cells, and the cells were screened with $1 \mu \mathrm{g} / \mathrm{ml}$ puromycin for 3-4 weeks after transfection for $48 \mathrm{~h}$.

For transfection, cells in the logarithmic growth phase were inoculated into 12 -well plates at $2.5 \times 10^{5}$ cells/well. Transfection was conducted with Ribo FECTTM CP reagent (RiboBio) according to the manufacturer's instructions in RPMI-1640 culture medium without serum and antibiotic. Six hours after transfection, we continued to culture the cells in the medium containing serum for at least $48 \mathrm{~h}$ before the subsequent experiments. The transfection efficiency was determined by quantitative real-time PCR (qRT-PCR).

\section{RNA extraction and qRT-PCR}

Total RNA was isolated with TRIzol reagent (Invitrogen, Carlsbad, CA, USA) and CDNA was generated using a RevertAid First Strand CDNA Synthesis Kit (Thermo Fisher Scientific, Waltham, MA, USA) according to manufacturer's guidelines. The reverse transcription reaction for miR-150-5p was performed using a Maxima $\mathrm{H}$ Minus First Strand CDNA Synthesis Kit (Thermo Fisher Scientific). qRT-PCR was performed using ChamQ ${ }^{\mathrm{TM}}$ Universal SYBR qPCR Master Mix (Vazyme Biotech, Nanjing, China). LncRNA and mRNA expression were normalized to GAPDH expression, and miRNA was normalized to U6 snoRNA. The sequences of the primers used are listed in Supplementary Table S2.

\section{CCK-8, Ki-67, Edu, and colony formation assays}

Transfected cells were seeded into a 96-well plate at a density of $5 \times 10^{4}$ cells $/ \mathrm{ml}$. Subsequently, $10 \mu \mathrm{l}$ of Cell Counting Kit-8 (CCK-8) solution (Bimake, Houston, TX, USA) was added to each well at the same time every day for 4 days. After a 3-h incubation, the absorbance was measured at $450 \mathrm{~nm}$ through a microplate reader (BioTek ELX800, Winooski, VT, USA).

Transfected cells were stained with the Ki-67 antibody (\#ab15580, Abcam) for $30 \mathrm{~min}$ in the dark. Cells were washed twice with $40 \mathrm{ml}$ phosphate-buffered saline $/ 0.5 \%$ bovine serum albumin, resuspended in $5 \mathrm{ml}$ phosphate-buffered saline/0.5\% bovine serum albumin, and stained with the viability marker 7-AAD on ice for $10 \mathrm{~min}$ in the dark. The analysis was performed using BD FACS Diva. Sorting was performed by using a MoFlo high-speed cell sorter (Beckman Coulter).

Ethynyl deoxyuridine (Edu) incorporation was used to assess cell proliferation with EdU Kit (RiboBio) according to the manufacturer's instructions. The cells were quantified with a fluorescence microscope (Olympus, Tokyo, Japan) and Image-Pro Plus 6.0 software.

For colony formation assays, cells $\left(3 \times 10^{3} /\right.$ well) were seeded in 12 -well culture plates with three wells in each group. The cells were resuspended in $0.33 \%$ agar and nutrition was performed twice a week by adding two drops of MDS cell cultures to the medium. After incubation for 14 days at $37^{\circ} \mathrm{C}$, images of the 12-well plate were taken under a microscope then scanned, and counted the colony numbers with ImageJ.

\section{Cell cycle and apoptosis assay}

For the cell cycle analysis, cells were fixed with $70 \%$ cold ethanol and stained with $0.1 \mathrm{mg} / \mathrm{ml}$ PI (Beyotime Biotechnology, Shanghai, China). Cell cycle distribution was analyzed by FACSCanto II flow cytometer (BD Biosciences San Diego, CA, USA). Apoptosis assay was performed with annexin V-FITC/PI Apoptosis Detection Kit (Vazyme Biotech) according to the manufacturer's instructions. FlowJo software (vX.0.7, Ashland, OR) was used to analyzed data

\section{FISH and subcellular fractionation of $\mathrm{BC} 200$}

To determine the subcellular location of BC200, locked nucleic acid-RNA fluorescence in situ hybridization (LNA-FISH) was performed with a FISH kit (RiboBio) according to the manufacturer's protocol. LNA fluoresceinlabeled probes against 18 S rRNA, U6 snoRNA, and BC200 were designed and synthesized by Ribo. Fluorescence signals were scanned using a Leica SP5 II scanning confocal microscope (Leica, Bannockburn, USA). A nucleus

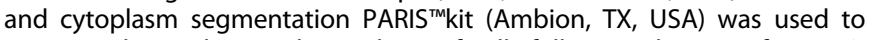
segment the nucleus and cytoplasm of cells following the manufacturer's instructions.

\section{Construction of the IncRNA-miRNA-mRNA regulatory network} The independent cohorts of primary MDS data of GSE114869 were downloaded from the Gene Expression Omnibus (GEO) database [13]. The relationship between differentially expressed IncRNAs (DElncRNAs) and DEmiRNAs was explored by the starBase v3.0 database based on CLIP-seq data research and the miRcode database based on the GENCODE database [22]. TargetScan, miRcode, and MiRanda were further used to determine the interactions between DEmiRNAs and DEmRNAs. Finally, according to the interactions between IncRNAs, miRNAs, and mRNAs, a ceRNA regulatory network was constructed and visualized by Cytoscape 3.6.1.

\section{Luciferase reporter assay}

To evaluate promoter activity, BC200 promoter was cloned into GV238 vector. Then, HEK293T cells was cotransfected with GV238-BC200 and MYB siRNAs or control siRNA with Lipofectamine 3000 (Invitrogen). For 3'-UTR luciferase reporter assays, luciferase reporters were synthesized by cloning wild-type (wt) or mutant $(\mathrm{mt})$ BC200 3'-UTR sequence containing the miR150-5p binding site into GV272 vector. Cells were cotransfected with miR$150-5 \mathrm{p}$ mimics or control vector and GV272-BC200 or GV272-BC200-mt (miR-150-5p) using Lipofectamine 3000. Likewise, GV272-MYB or GV272MYB-mt (miR-150-5p) was cotransfected with miR-150-5p mimics or 
control vector into HEK293T cells. After $48 \mathrm{~h}$, firefly and Renilla luciferase activities were examed using the Dual-Luciferase Reporter Assay System (Promega, WI, USA) according to the manufacturer's instructions. Luciferase activities were normalized to Renilla luciferase.

\section{ChIP assay}

Pierce $^{T M}$ Magnetic chromatin immunoprecipitation (ChIP) Kit (Thermo Fisher Scientific) was used to perform ChIP assays. Briefly, MDS-L cells were crosslinked with $1 \%$ formaldehyde. Chromatin was isolated and immunoprecipitated with an MYB antibody (\#05-175, Sigma-Aldrich, St Louis, MO, USA). Immunoprecipitated DNA was washed and eluted according to the manufacturer's instructions. qRT-PCR was conducted to analyze the eluted DNA. The sequences of the primers used in ChIP assays are listed in Supplementary Table S3.

\section{RNA immunoprecipitation (RIP) assay}

RIP was performed with a Magna RIP RNA-Binding Protein Immunoprecipitation Kit (Millipore, Billerica, MA, USA). In brief, MDS-L cells were collected and lysed in RIP buffer. Next, $100 \mu \mathrm{L}$ cells containing magnetic beads were conjugated with human anti-Ago2 antibody (Abcam, Cambridge, MA, USA) or negative control (normal mouse lgG, Millipore). Finally, the immunoprecipitated RNA was detected by qRT-PCR to measure BC200 and miR-150-5p levels in the precipitates.

\section{RNA pull-down assay}

A DNA fragment containing the full-length $\mathrm{BC} 200$ sequence or a negative control sequence was PCR amplified using T7 RNA polymerase (Roche, Basel, Switzerland). The resulting plasmid DNA was linearized using the restriction enzyme Xhol. Biotin-labeled RNA was reverse transcribed using Biotin RNA Labeling Mix (Roche) and T7 RNA polymerase (Takara Biomedical Technology). The products were treated with RNase-free DNase I (Roche) and purified with an RNeasy Mini Kit (Qiagen, MD, USA), with the resulting RNA used for real-time $\mathrm{PCR}$ assays. Moreover, the products were treated with Ago2 antibody (\#03-110, Sigma-Aldrich) to detect cell lysates utilizing the sample pulled down by biotinylated BC200 and random probe.

\section{Western blot analysis}

MDS cells were lysed with RIPA buffer (Vazyme Biotech) in the presence of a protease inhibitor cocktail (Vazyme Biotech, Nanjing). $50 \mu \mathrm{g}$ of protein extracts were boiled and subject to 10\% SDS-PAGE gel and then transferred to nitrocellulose membranes. The membranes were blocked by $5 \%$ non-fat dry milk (Bio-Rad, CA, USA) and incubated with mouse anti-human MYB (Sigma-Aldrich) and mouse anti-human GAPDH primary antibodies (\#sc-32233, Santa Cruz Biotechnology, Dallas, TX, USA) (the dilutions of primary antibodies is $1: 1000$ ) at $4{ }^{\circ} \mathrm{C}$ overnight. The HRP-conjugated secondary antibodies were used at 1:3000 dilution for $1.5 \mathrm{~h}$ at room temperature. Signals were detected by ECL HRP substrate (Millipore).

\section{Tumorigenicity assays in vivo}

All animal experiments were approved by the Animal Care and Use Committee of the Third Xiangya Hospital of Central South University (Changsha, China). All NOD-Prkdcem26Cd52Il2rgem26Cd22/NjuCrl (NCG) female mice, 4-6 weeks, were purchased from the Shanghai Lab Animal Research Center (Shanghai, China) and randomly divided into three groups (sample size: four mice per group). And investigators were blinded to the randomization. The right upper backs of NCG mice were injected subcutaneously with $5 \times 10^{6}$ cells stably transfected with sh-BC200 mixed with $0.2 \mathrm{ml} \mathrm{RPMI-1640} \mathrm{medium.} \mathrm{The} \mathrm{tumor} \mathrm{volume} \mathrm{for}$ each mouse was measured every 2 days. On 26th day, the tumorbearing mice were sacrificed, and the size of the tumors was measured and stored.

\section{Intravenous MDS model of NCG mice}

The female NCG mice, 4-6 weeks, were randomly divided into three groups (sample size: three mice per group) and investigators were blinded to the randomization. SKM-1-vector (transfected with empty vector) and SKM-1 sh-BC200-1/2 (transfected with shRNA targeting BC200) cells (6x $10^{6}$ cells per animal) were separately intravenously injected into the tail vein of NCG mice. After 1 month, the mice were sacrificed, and BM, peripheral blood, and tissue samples were collected.

\section{Fluorescence-activated cell sorting (FACS)}

To analyze BM samples of intravenous MDS mice by flow cytometry, the $B M$ cells were stained with the following anti-human antibodies: CD34-APC (\#343608, BD Pharmingen), CD45-APC-Cy (\#304014, BD Pharmingen), CD71-PE (\#334106, BD Pharmingen), glycophorin A (GPA)-BV421 (\#562938, Biolegend), CD36-BV605 (\#563518, Biolegend), CD38-FITC (\#356610, Biolegend), anti-IL-3R (CD123)-PE-Cy7 (\#25-1239-42, Invitrogen) for $30 \mathrm{~min}$ in the dark. Cells were washed twice with $40 \mathrm{ml}$ phosphatebuffered saline/0.5\% bovine serum albumin, resuspended in $5 \mathrm{ml}$ phosphate-buffered saline $/ 0.5 \%$ bovine serum albumin, and stained with the viability marker 7-AAD on ice for $10 \mathrm{~min}$ in the dark. The analysis was performed using BD FACS Diva. Sorting was performed by using a MoFlo high-speed cell sorter (Beckman Coulter).

\section{Peripheral blood cells count}

After inoculating the NCG mice with SKM-1 cells via the tail vein for 30 days, blood samples were collected into ethylenediaminetetraacetic acid-coated tubes and full blood counts were determined on an Advia 120 automated hematology analyzer.

\section{Immunohistochemical (IHC)}

The paraffin-embedded sections were from tumors and tissues of mice used for evaluating the expression of MYB and Ki-67 protein. Immunohistochemical staining used a streptavidin-peroxidase method according to the manufacturer's introduction.

\section{Bioinformatics analysis}

Two primary MDS datasets cohorts GSE114869 and GSE99095 were downloaded from the GEO database. GSE114869 has 300 primary MDS samples and 20 normal MDS samples; GSE99095 comes from the singlecell RNA sequencing for five primary MDS samples and four normal MDS samples. GSE114869 and GSE99095 gene expression profile data were analyzed by means of Significant Analysis of Microarray (SAM) software.

\section{Statistical analysis}

Student's $t$-test was used to determine the significance of the differences between the control and the experimental groups using the SPSS 22.0 statistical software and GraphPad Prism 8.0 (GraphPad software). Values were shown as mean \pm SD for three independent experiments. $P<$ 0.05 was considered to indicate statistical significance. The correlation analysis was performed using Pearson's correlation test. $P<0.05$ was considered to indicate statistical significance.

\section{RESULTS}

Knockdown of BC200 inhibits the proliferation of MDS cells To explore the role of BC200 in the proliferation of MDS cells, we selected two shRNA with the most effective interference effect and confirmed the knockdown effect in SKM-1 and MDS-L cells (Fig. 1A). Cell proliferation was analyzed by CCK-8, Edu incorporation, colony formation assays, and FACS analysis. Knockdown of BC200 significantly suppressed MDS cell viability, which was detected by CCK-8 and Ki-67 (Fig. 1B and Fig. S1A). Edu incorporation and colony formation assays also indicated that knockdown of BC200 also inhibited the malignant proliferation ability of SKM-1 and MDS-L cells compared to the paired negative control shRNA (Fig. 1C, D). Notably, we explored whether knockdown of BC200 suppressed the proliferation of MDS-L and SKM-1 cells by inducing cell cycle arrest at G0/G1 phase rather than cell apoptosis (Fig. $1 \mathrm{E}-\mathrm{G}$ and Fig. S1B, C). The results indicated that knockdown of $\mathrm{BC} 200$ by two BC200 siRNAs inhibited the proliferation of primary bone marrow mononuclear cells (BMMCs) obtained from MDS patients and cultured in vitro (Fig. 1H-J).

BC200 acts as a molecular sponge for miR-150-5p in MDS cells As indicated in previous reports, the location of IncRNAs frequently determines their functions [23]. In this study, to investigate the mechanisms by which BC200 functions as a carcinogen in MDS, we first determined its location in SKM-1 cells. 

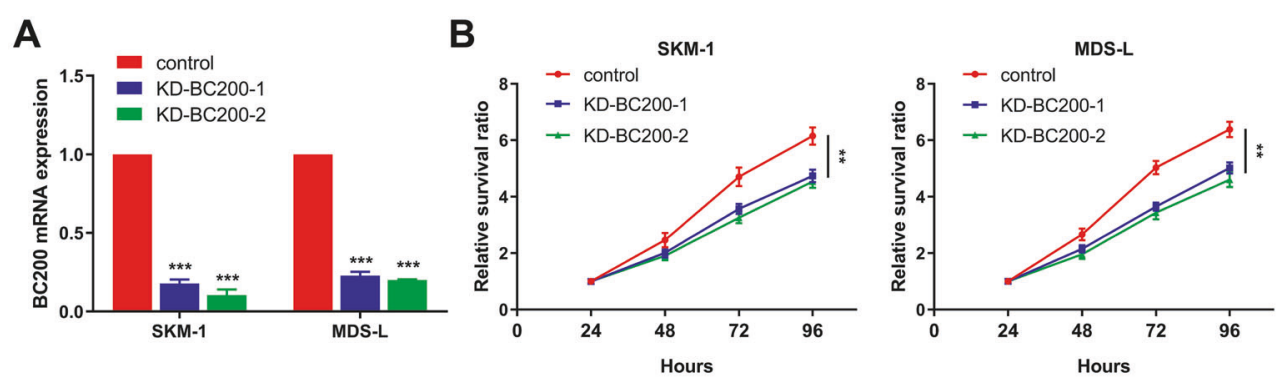

C
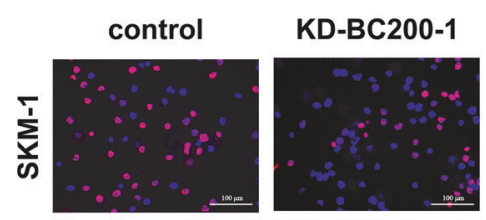

KD-BC200-2
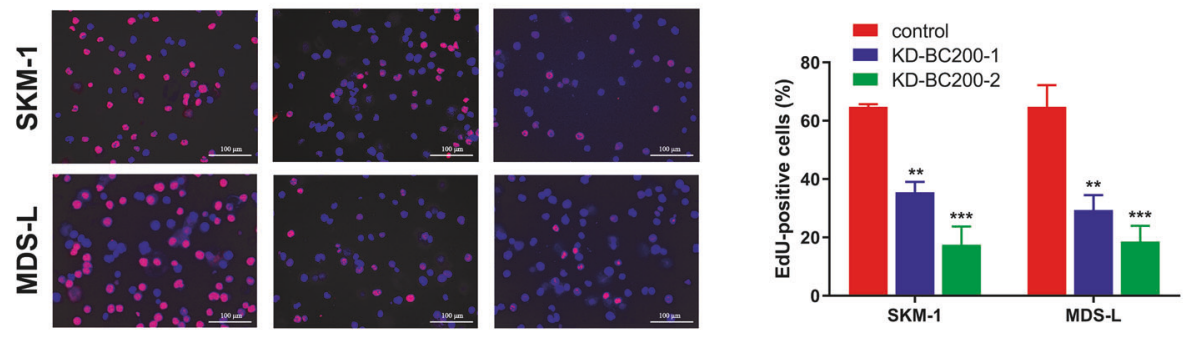

D

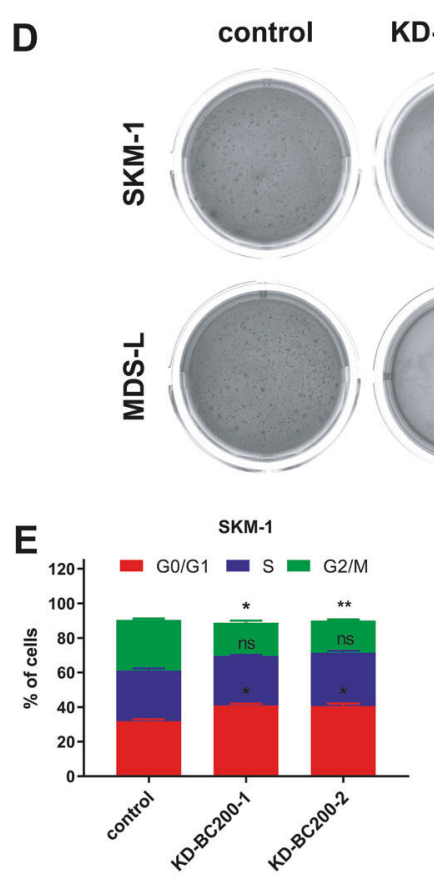

KD-BC200-1 KD-BC200-2
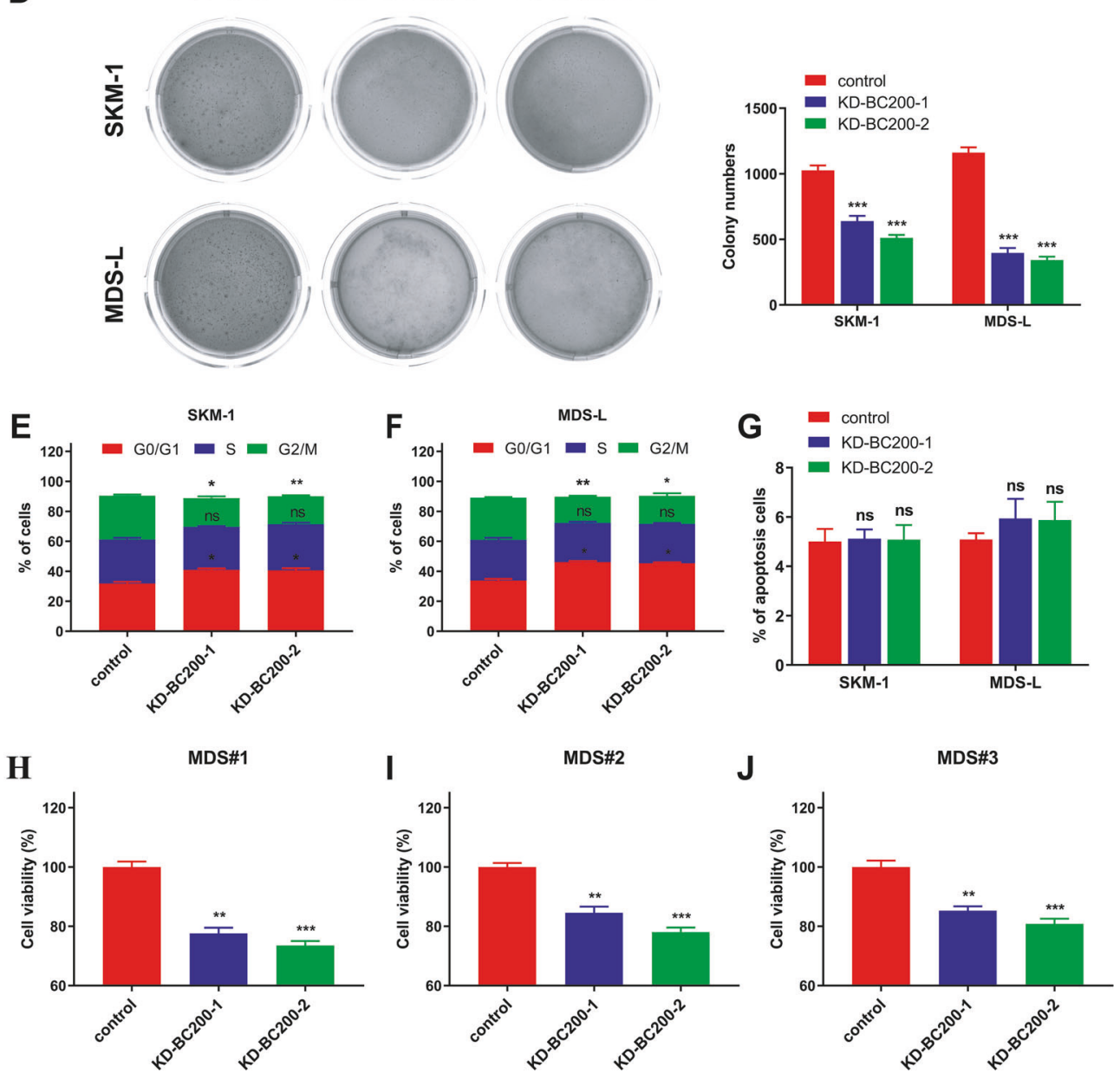

Fig. 1 Knockdown of BC200 inhibits the proliferation of MDS cells. A In SKM-1 and MDS-L cells, the transcriptional level of BC200 was downregulated by two shRNAs. B-D MDS cells proliferation was detected by CCK-8, EdU incorporation (Scale bar, $100 \mu \mathrm{m}$ ) and colony formation assays. It revealed that BC200 knockdown significantly suppressed SKM-1 and MDS-L cell proliferation. E, F Knockdown of BC200 led to G0/G1 arrest in both SKM-1 and MDS-L cells. G Apoptosis assay showed that the percentage of apoptotic MDS cells was not affected by BC200 knockdown. H-J Human primary BMMCs were treated with BC200 siRNAs for $72 \mathrm{~h}$, and cell proliferation was analyzed by the CCK-8 assay. ${ }^{*} p<0.05,{ }^{* *} p<0.01,{ }^{* * *} p<0.001$, ns, not significant. 
A
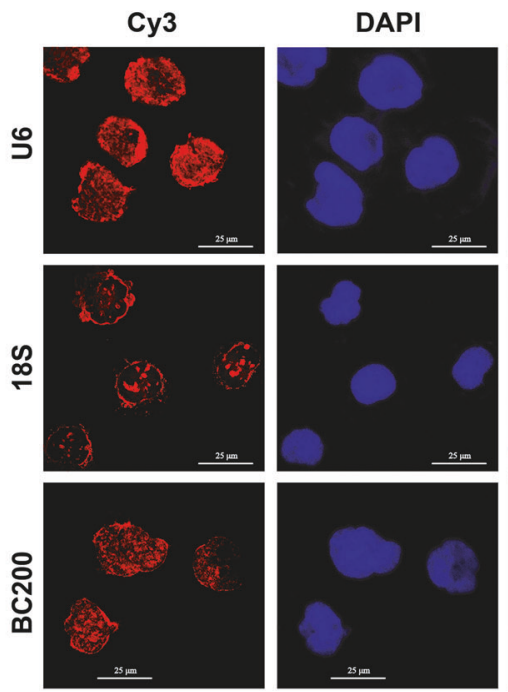

B

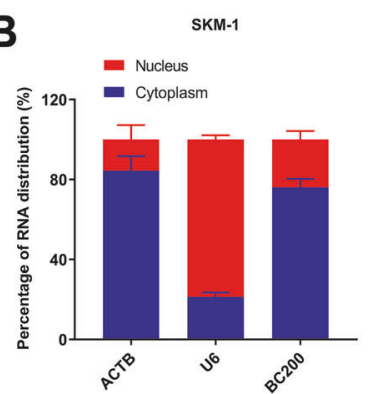

$\mathbf{F}$
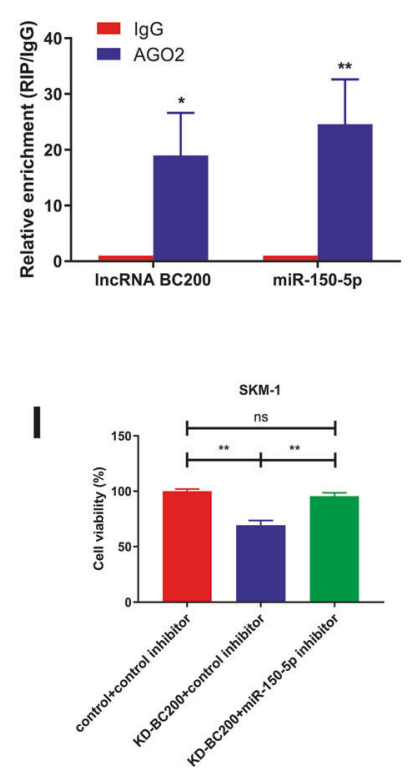

G
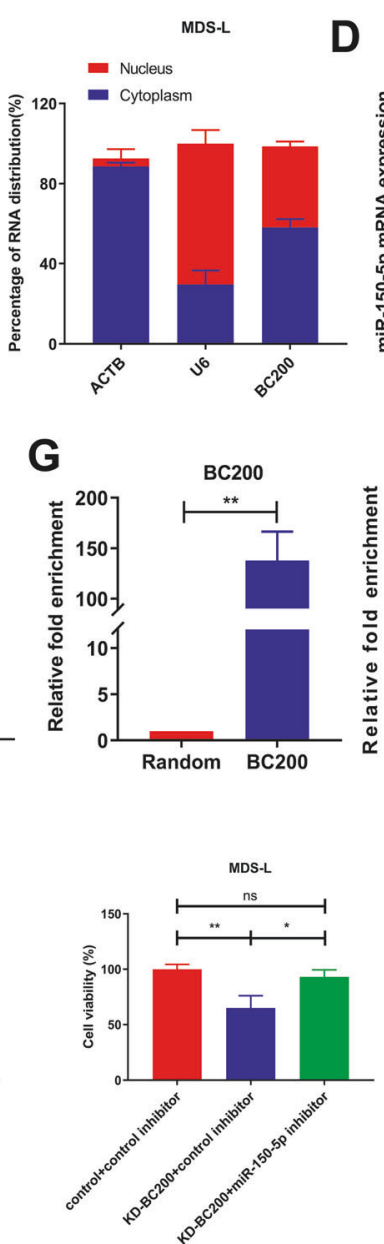

As the FISH and subcellular fractionation data show, BC200 was mainly located in the cytoplasm of SKM-1 cells, indicating that BC200 plays a regulatory role in MDS cells through the ceRNA network (Fig. 2A, B). We further analyzed the DElncRNAs in the RNA sequencing data of a GEO dataset (GSE114869) and generated a ceRNA network diagram (Fig. S2A). We separately analyzed the ceRNA regulatory network of $\mathrm{BC} 200$ and predicted three miRNAs (miR-150-5p, miR-181b, and miR-590-3p) containing
C

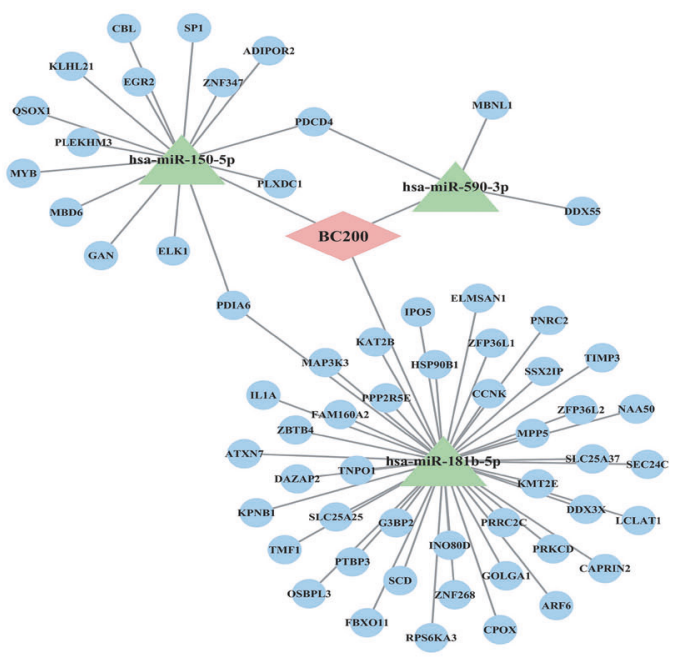

E $\begin{aligned} & \text { AR-150-5p G T G A C C A T G T T C C C A A C C C T C T } \\ & \text { BC200-mut } \\ & \text { A G G G A G G T A A G A G G C T T T C T T }\end{aligned}$

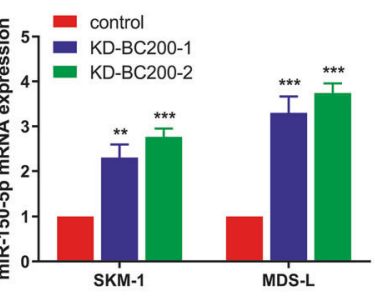

- control mimics

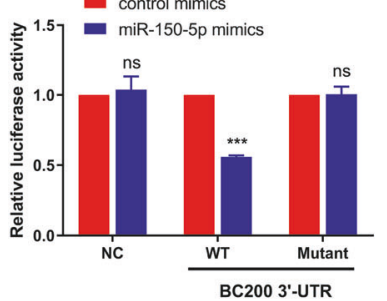

H
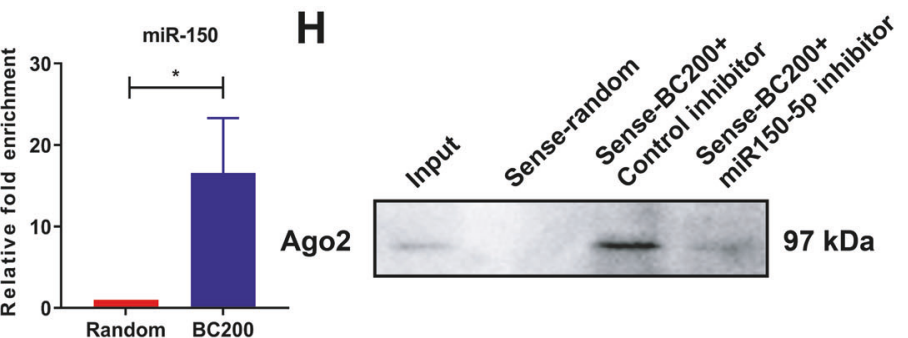
Fig. 2 BC200 acts as a molecular sponge for miR-150-5p in MDS cells. A RNA FISH assays for BC200 in SKM-1 cells. Stain the nucleus with DAPI. Scale bar, $25 \mu \mathrm{m}$. B BC200, U6, and ACTB expression in the RNA extracted from the cytoplasm and nucleus of MDS cells by qRT-PCR. C The ceRNA regulatory network of BC200 and three miRNAs containing the binding site of BC200. D Transcriptional level of miR-150-5p was detected in sh-BC200 MDS cells. E Upper: complementary sequence between miR-150-5p and BC200-wt. The putative binding sites of miR150-5p in BC200-mt. Lower: Luciferase activity was measured in HEK293T cells cotransfected with miR-150-5p mimics and wt or mt BC200 vector. F RIP assays of the enrichment of Ago2 on BC200 and miR-150-5p relative to IgG in MDS-L cells. The relative expression levels of BC200 and miR-150-5p were detected by qRT-PCR. G Detection of BC200 or miR-150-5p by using qRT-PCR in the sample pulled down by biotinylated BC200 and Random probe. Input was used for normalization. H The correlation among BC200, miR-150-5p, and Ago2 was determined by using Ago2 antibody to detect cell lysates utilizing the sample pulled down by biotinylated BC200 and Random probe. I CCK-8 assay rescue experiment showed that cell proliferation reduced by si-BC200 could be increased by miR-150-5p inhibitor in MDS cells. J CCK-8 assay rescue experiment showed that cell proliferation stimulated by overexpression of BC200 could be suppressed by miR-150-5p mimics in MDS cells. ${ }^{*} p<0.05,{ }^{* *} p<0.01,{ }^{* * *} p<0.001$, ns, not significant.

150-5p were associated with the RNA-induced silencing complex (RISC), RIP assays were performed utilizing an antibody against Ago2 (the core component of the RISC). It revealed that both BC200 and miR-150-5p were drastically enriched in Ago2 pellets compared with IgG immunoprecipitates in MDS-L cells (Fig. 2F), suggesting that BC200 was physically present in the Ago2-based miRNA-induced repression complex and was associated with miR$150-5 \mathrm{p}$. Subsequently, the biotin-labeled pull-down results showed a significant amount of BC200 and miR-150-5p in the BC200 pull-down pellet compared with that observed in the control group as measured by qRT-PCR (Fig. 2G, H). These data indicated that BC200 could negatively regulate miR-150-5p expression in MDS cells.

To ascertain whether miR-150-5p could affect MDS cell proliferation, we transfected miR-150-5p mimics and miR-150-5p inhibitors in MDS cells. The CCK-8 results showed that miR-150-5p mimics suppressed MDS cell proliferation after transfection compared to the control mimics (Fig. S2D). In contrast, the miR150-5p inhibitor promoted MDS cell proliferation after transfection (Fig. S2E). Furthermore, overexpression of miR-150-5p significantly suppressed MDS cell viability detected by Ki-67 (Fig. S2F). Notably, miR-150-5p mimics suppressed the proliferation of MDS-L and SKM-1 cells by inducing cell cycle arrest at $S$ phase and promoting cell apoptosis (Fig. S2G, H).

Moreover, inhibition of miR-150-5p rescued the suppressive effect of BC200 knockdown by siRNA on MDS cells (Fig. 2l), while overexpression of miR-150-5p attenuated the increased proliferation abilities of MDS cells overexpressing BC200 (Fig. 2J). Thus, these results strongly suggested that the oncogenic effect of BC200 on MDS was partially mediated by miR-150-5p.

\section{MYB is a direct target of miR-150-5p in MDS cells}

To explore into the molecular mechanisms for miR-150-5p suppressing MDS cell proliferation, starBase v3.0 and TargetScan bioinformatic analyses were conducted to forecast the putative targets of miR-150-5p. Among the targets, MYB, which ranked number one, had a conserved miR-150 binding site and was identified as a potential target of miR-150-5p (Fig. 3A). Previously, MYB was reported as a direct target of miR-150-5p in hepatocellular carcinoma $[24,25]$. Our results showed that overexpression of miR-150-5p markedly downregulated MYB at the mRNA and protein levels, while inhibition of miR-150-5p upregulated the expression of MYB in SKM-1 and MDS-L cells (Fig. 3B-E). To confirm whether MYB is a target gene for miR-150-5p, a luciferase reporter assay was performed. In HEK293T cells, overexpression of miR-150-5p reduced the luciferase activity of GV272-MYB but did not influence that of GV272-MYB-mt (Fig. 3F). Moreover, we examined the function of MYB in MDS cell proliferation. As shown in Supplementary Fig. S3A-D, silencing MYB in MDS cells by using two siRNAs significantly suppressed MDS cell proliferation.

To further verify that MYB is a functional target of miR-150-5p and is responsible for promoting cell proliferation, miR-150-5p mimics/inhibitor and MYB plasmid/siRNA were introduced alone or simultaneously into MDS cells. Overexpression of MYB restored the inhibitory effect of miR-150-5p mimics on MDS cell proliferation (Fig. 3G). Conversely, MYB knockdown attenuated the miR150-5p inhibitor-induced proliferation of MDS cells (Fig. $3 \mathrm{H}$ ). Hence, these findings demonstrated that miR-150-5p regulated the proliferation of MDS cells by targeting MYB expression.

\section{BC200 promotes the malignant proliferation of MDS cells via} the miR-150-5p/MYB axis

It was further confirmed that $\mathrm{BC} 200$ promoted the proliferation of MDS cells via the miR-150-5p/MYB axis, and BC200 negatively regulated miR-150-5p. We found that knockdown of $B C 200$ reduced both MYB mRNA and protein levels (Fig. 4A, B), while overexpression of BC200 significantly increased the mRNA and protein levels of MYB (Fig. 4C, D). Furthermore, the upregulation of MYB induced by BC200 overexpression was abrogated by miR150-5p mimics, and similarly, the reduction in MYB levels induced by knockdown of BC200 by siRNA was reversed by the miR-150-5p inhibitor in both SKM-1 and MDS-L cells (Fig. 4E, F). On the other hand, overexpression of MYB completely reversed the cell proliferation-inhibiting effects of BC200 siRNA, while the increase in MDS cell proliferation by $\mathrm{BC} 200$ overexpression was abolished by knockdown of MYB (Fig. 4G, H). Thus, our findings indicated that $\mathrm{BC} 200$ promoted MDS malignant proliferation through the miR-150-5p/MYB axis.

MYB transcriptionally regulates BC200 expression in MDS cells To understand the potential mechanism of the abnormally high expression of BC200 in MDS, we used the ChIP-seq data from the public UCSC Genome Browser (http://genome.ucsc.edu/index. html) to identify BC200 potential transcription factors. Interestingly, the transcription factor MYB had been identified as potentially binding to the upstream regulatory region of the BC200 gene (Fig. S4). To verify MYB could regulate BC200 in MDS, we proved that knockdown of MYB significantly reduced BC200 expression (Fig. 5A). To further determine the transcriptional activity of MYB on the BC200 gene promoter, GV238-BC200 and MYB siRNAs were cotransfected into cells. As shown in Fig. 5A, knockdown of MYB markedly reduced BC200 promoter activity. Furthermore, it has been predicted that the transcription factor MYB has two binding sites on the BC200 promoter, "TGCAACC GAG" (Site 1) and "CGCATCTGTA" (Site 2) using bioinformatics analyses (JASPAR, ifti.org) (Fig. 5B). ChIP assays demonstrated the significant enrichment of MYB on binding Site 1 of BC200 promoter (Fig. 5C). In further studies of the effect of MYB on BC200 transcription in MDS, we found that knockdown of MYB significantly reduced BC200 expression in SKM-1 and MDS-L cells (Fig. 5D). More importantly, rescue assays were conducted to determine whether MYB promotes malignant proliferation through BC200 in MDS cells. We found that MYB suppression decreased cell proliferation, whereas BC200 overexpression markedly rescued this phenotype (Fig. 5E). In contrast, MYB overexpression increased cell proliferation, whereas BC200 suppression markedly rescued this phenotype (Fig. 5F). Taken together, these results illustrated that the BC200/miR-150- 
A
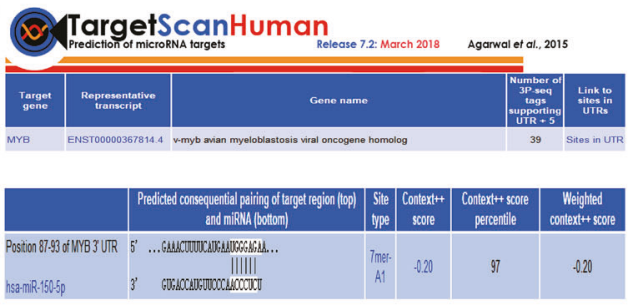

B

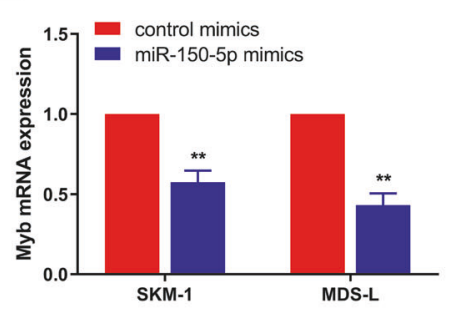

C

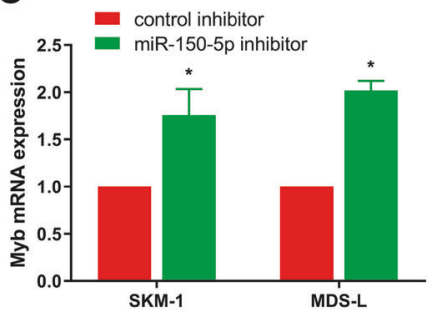

D
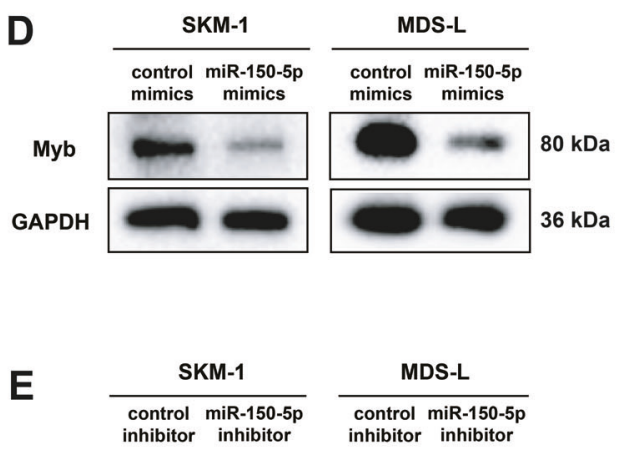

Myb inhibitor inhibitor
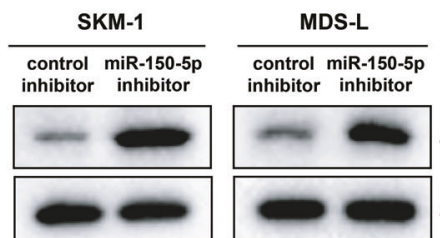

GAPDH

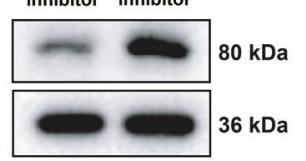

F

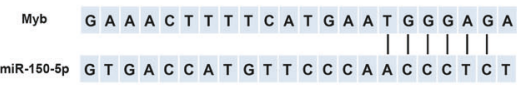

$\underset{\substack{\text { Myb-mut } \\ \text { miR-150-5p) }}}{\text { G A A A C T T T T C A T G A A A C C C T C T }}$

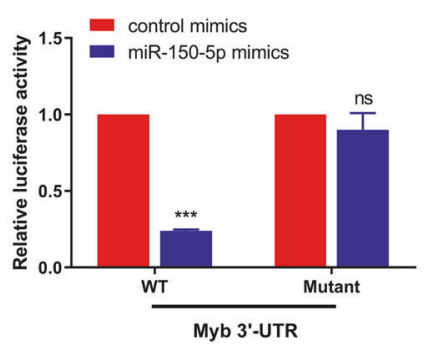

G

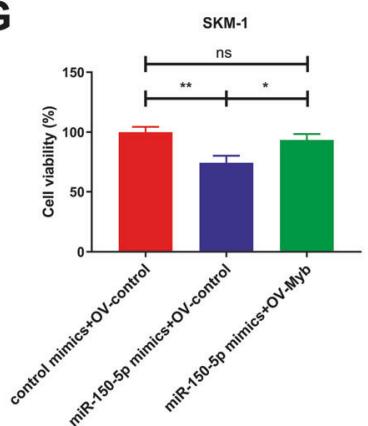

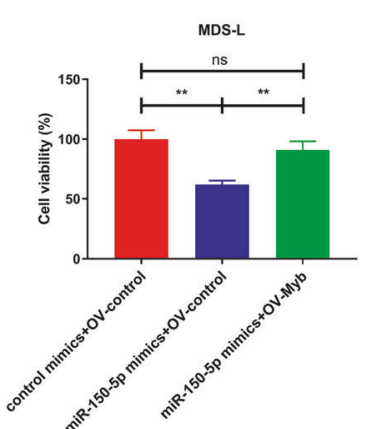

H

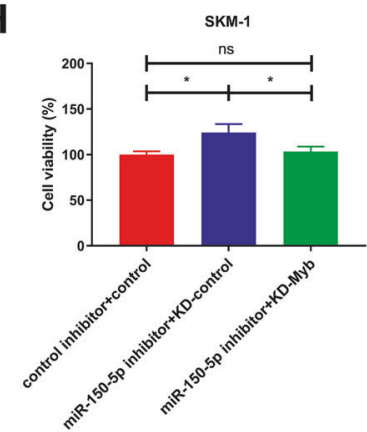

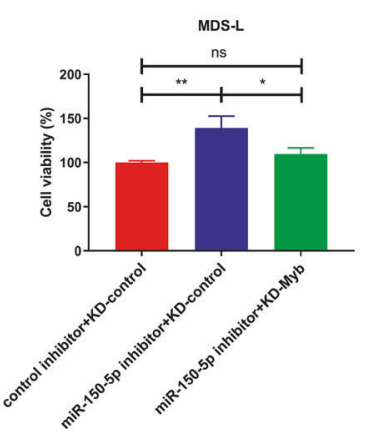

Fig. 3 MYB is a direct target of miR-150-5p in MDS cells. A MYB was the top candidate target containing the complementary site for the seed region of miR-150-5p in the TargetScan database. B, C SKM-1 and MDS-L cells were transfected with miR-150-5p mimics or inhibitors for $48 \mathrm{~h}$, and MYB mRNA levels were detected by qRT-PCR. D, E MYB protein expression in both SKM-1 and MDS-L cells transfected with miR-150$5 \mathrm{p}$ mimics or inhibitor for $48 \mathrm{~h}$ was detected by western blotting. $\mathbf{F}$ Upper: putative MYB binding sites on the promoter region of miR-150-5p and their corresponding mutant binding sites. Lower: luciferase activity in HEK293T cells cotransfected with miR-150-5p or control mimics and luciferase reporters containing the wt or mt MYB 3'-UTR. G CCK-8 assay detected the effect of MYB overexpression and miR-150-5p mimics on SKM-1 and MDS-L cell proliferation activity after $72 \mathrm{~h}$. H CCK-8 assay detected the effect of MYB knockdown and miR-150-5p inhibitor on SKM1 and MDS-L cell proliferation activity after $72 \mathrm{~h} .{ }^{*} p<0.05,{ }^{* *} p<0.01,{ }^{* * *} p<0.001$, ns, not significant.

5p/MYB positive feedback loop promotes malignant proliferation in MDS cells.

\section{The BC200/miR-150-5p/MYB loop promotes tumor growth in vivo}

We next explored the pro-proliferative role of the BC200/miR-150$5 \mathrm{p} / \mathrm{MYB}$ loop in vivo. First, subcutaneous xenografts were established in the flanks of NCG mice using sh-BC200-1/2 cells and sh-control cells. The volume (Fig. 6A, B) and weight (Fig. 6C) of BC200-silenced tumors were markedly smaller than those of tumors formed by control cells, while the mouse body weights were not significantly different between the BC200-silenced groups and the control group (Fig. 6D), indicating that sh-BC200 had no obvious side effects on the mice. In addition, the immunohistochemical staining results showed that both Ki-67 and MYB were expressed at low levels in BC200-silenced tumor tissues (Fig. 6E). qRT-PCR data showed that the mRNA levels of BC200 and MYB were downregulated, while miR-150-5p was upregulated in BC200-silenced tumor tissues compared with control tissues (Fig. 6F-H). Collectively, these results demonstrated that the BC200/miR-150-5p/MYB loop promoted the tumor growth of MDS cells in vivo.

Subsequently, we established the intravenous MDS mouse model by injecting sh-BC200-1/2 cells and sh-control cells. BC200 knockdown markedly reduced $\mathrm{CD} 123^{+} \mathrm{CD} 34^{+}$cells, which was related to the malignant clonal cells with aberrant differentiation, excessive proliferation, and decreased apoptosis in MDS (Fig. 61 and Fig. S5A). $\mathrm{CD} 36^{+}$and $\mathrm{CD} 38^{+} \mathrm{BM}$ cells were not significantly different between the $\mathrm{BC200}$-silenced groups and the control group, suggesting that there was no significant change in 
A

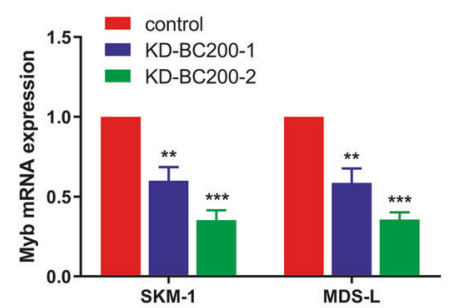

C

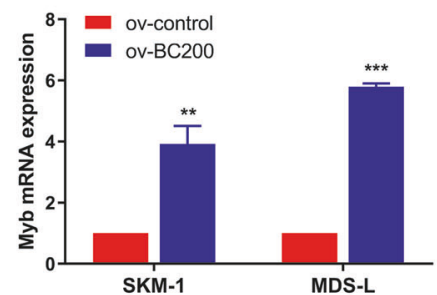

B

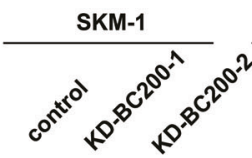

Myb
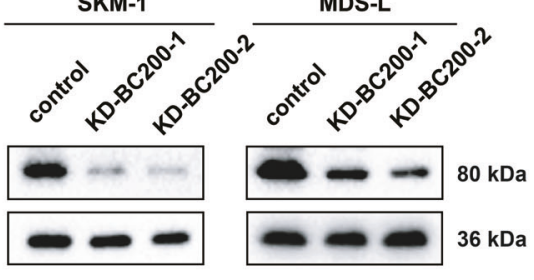

D

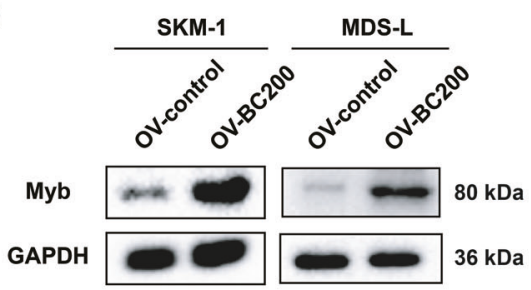

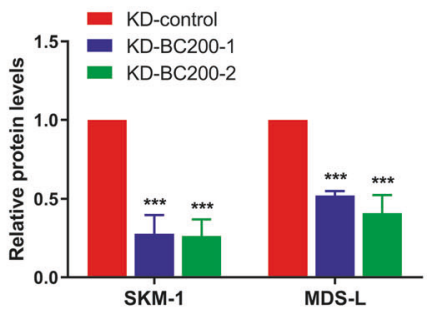

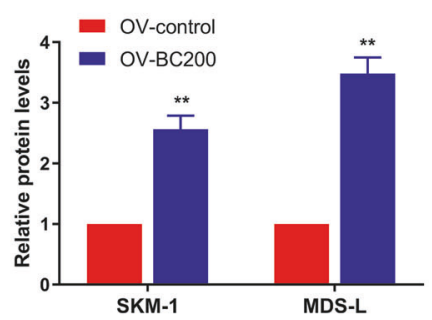

E

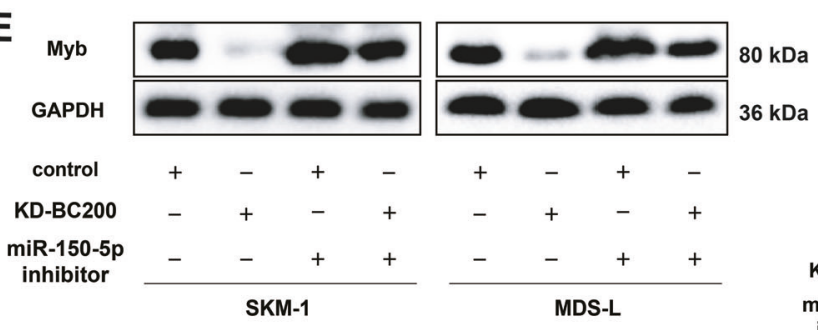

F

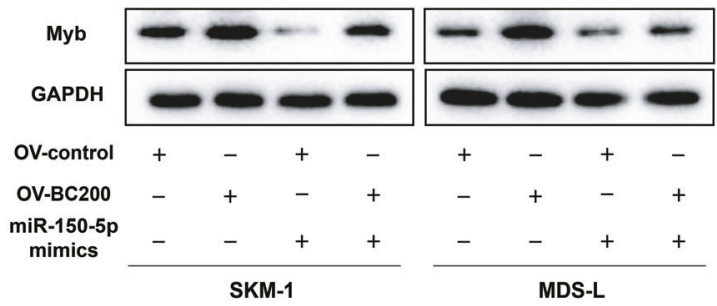

G
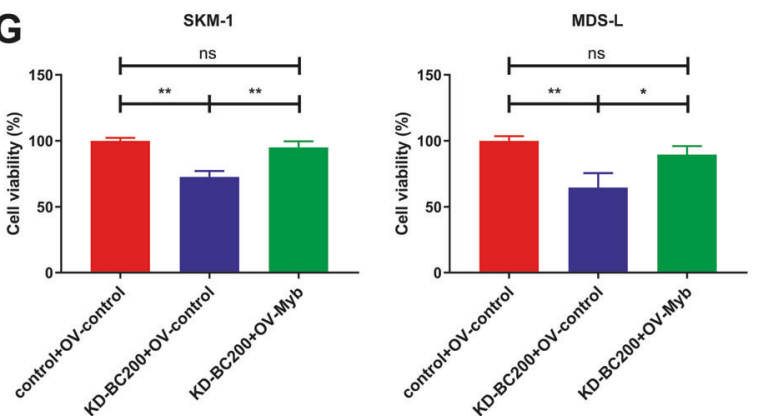
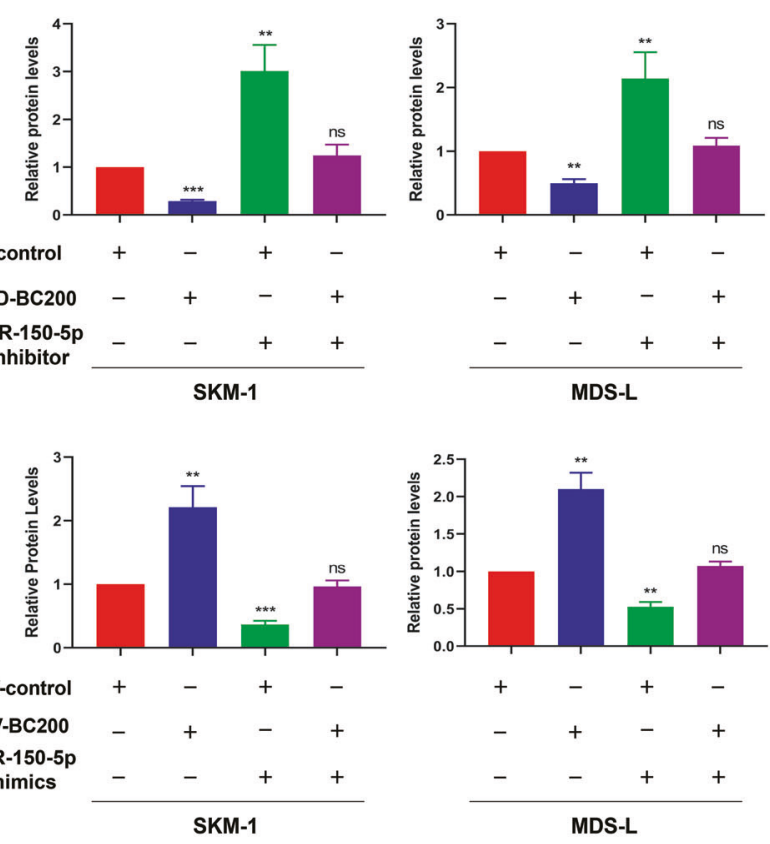

H

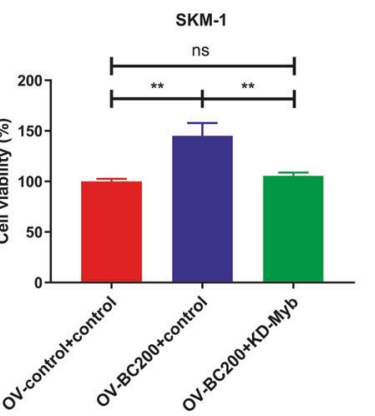

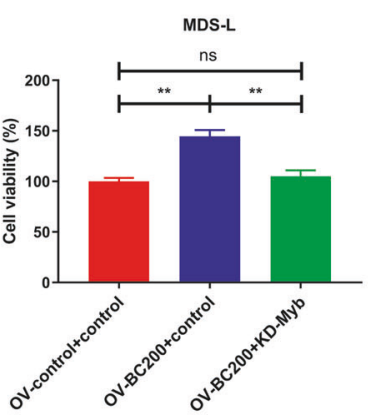

Fig. 4 BC200 promotes the proliferation of MDS cells via the miR-150-5p/MYB axis. A The mRNA levels of MYB were measured in sh-BC200 MDS cells. B The protein expression levels of MYB were measured in MDS cells transfected with two different BC200 shRNAs. C, D qRT-PCR and western blotting were used to measure the MYB mRNA and protein levels in SKM-1 and MDS-L cells transfected with BC200 plasmid (ovBC200) for $48 \mathrm{~h}$. E, F Western blot analysis of MYB in MDS cells cotransfected with miR-150-5p mimics and BC200 siRNA or miR-150-5p inhibitors and BC200 plasmid for 48 h. G CCK-8 assay indicated the effect of BC200 overexpression and MYB suppression on SKM-1 and MDS-L cell proliferation activity after $72 \mathrm{~h}$. H CCK-8 assay indicated the effect of BC200 knockdown and MYB upregulation on SKM-1 and MDS-L cell proliferation activity after $72 \mathrm{~h} .{ }^{*} p<0.05,{ }^{* *} p<0.01,{ }^{* * *} p<0.001, \mathrm{~ns}$, not significant. 
A

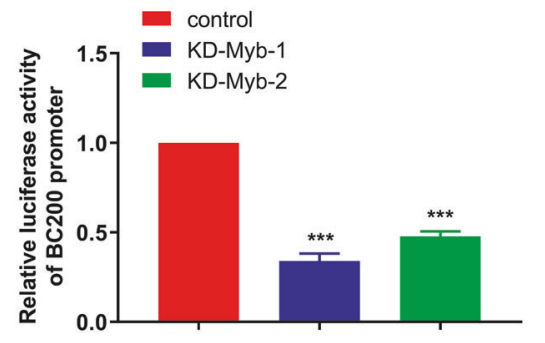

D

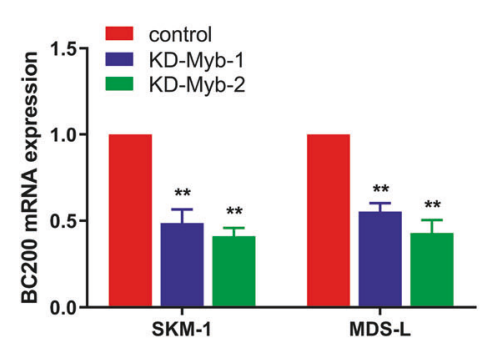

B

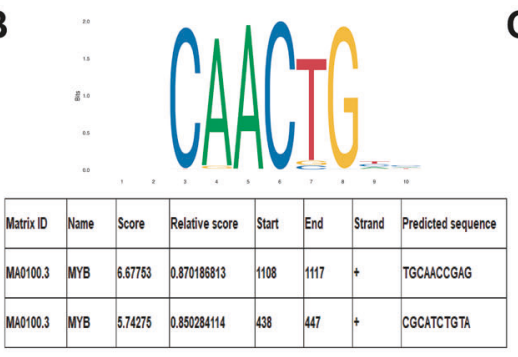

$\mathbf{E}$

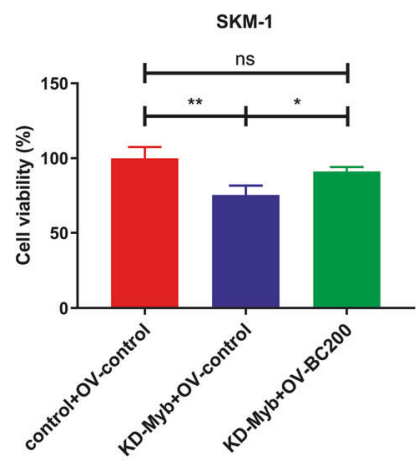

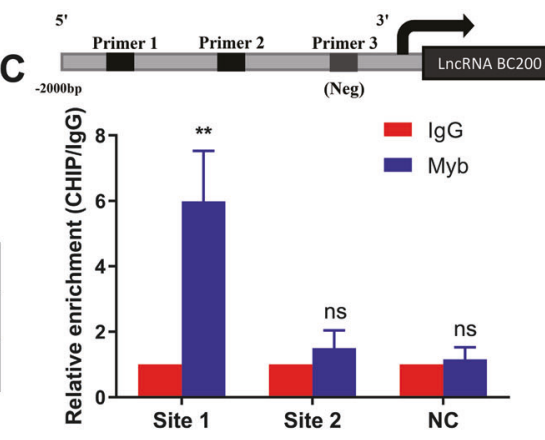

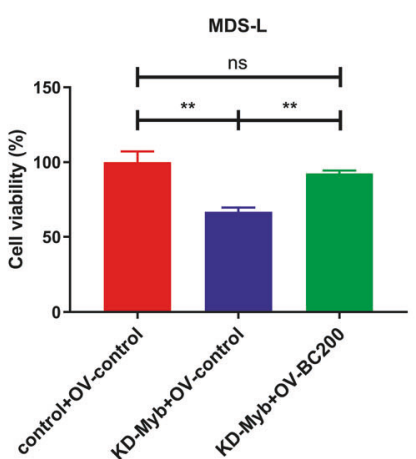

$\mathbf{F}$
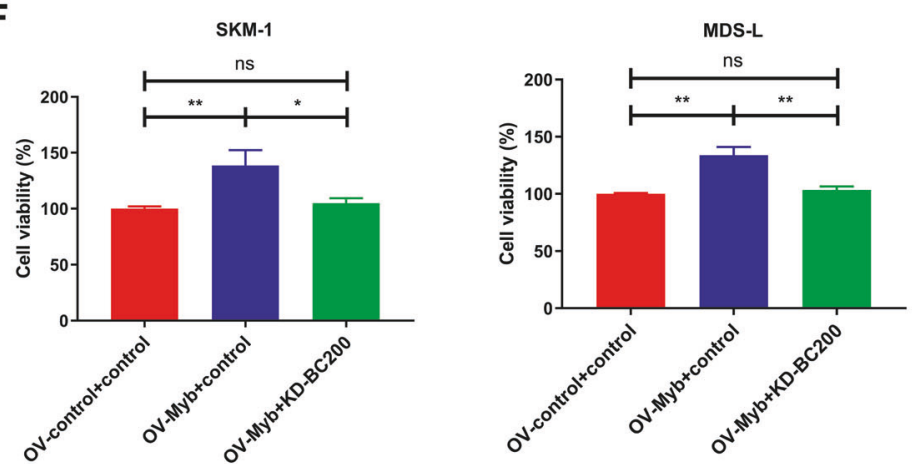

Fig. 5 MYB transcriptionally regulates BC200 expression in MDS cells. A HEK293T cells were cotransfected with GV238-BC200 and MYB siRNAs for $48 \mathrm{~h}$. B The MYB binding sitein BC200 predicted by JASPAR matrix models. C Upper: putative MYB binding sites BC200 promoter region and design of the indicated primers. Lower: ChIP assays of the enrichment of MYB on the BC200 promoter relative to control lgG in MDS-L cells. D The expression level of BC200 was detected in MDS cells transfected with two different MYB siRNAs. E, F SKM- 1 and MDS-L cells were treated with the indicated siRNAs or plasmids for $72 \mathrm{~h}$. The cell proliferative was measured by CCK-8 assay. ${ }^{*} p<0.05,{ }^{* *} p<0.01$, ${ }^{* * *} p<0.001$, ns, not significant.

granulocyte-monocyte and lymphoid lineage in BC200 knockdown BM cells (Fig. S5B-D). Moreover, Flow cytometry showed decreased human $\mathrm{CD}^{-} 1^{-} \mathrm{GPA}^{-}$, while increased $\mathrm{CD} 71^{-} \mathrm{GPA}^{+}$in BM cells of intravenous BC200-silenced groups, suggesting promoted differentiation from the undifferentiated progenitor cells to mature erythrocytes (Fig. S5E). Correspondingly, peripheral blood cells analysis further revealed that knockdown BC200 significantly increased red blood cells (RBCs), hemoglobin (HGB), hematocrit (HCT), and platelets (PLTs) and decreased white blood cells (WBCs) (Fig. $6 \mathrm{~J}$ ). In addition, the immunohistochemical staining results showed that the expression level of Ki-67 in the spleen of BC200-silencing group decreased slightly compared with the control group (Fig. S5F).

\section{Expression and clinical significance of the BC200/miR-150-5p/ MYB loop in MDS samples}

To determine the potential clinical significance of the BC200/miR$150-5$ p/MYB loop in MDS, we systematically analyzed the expression profiles of these genes in MDS samples versus normal samples using two published GEO datasets (GSE114869 and GSE99095) [26]. Analysis of the GEO data repository showed that the expression levels of both BC200 and MYB were dramatically higher, while miR$150-5 p$ was significantly lower in MDS samples than in normal samples (Fig. 7A-C and Fig. S6A). Furthermore, the expression of the BC200/miR-150-5p/MYB loop was detected by qRT-PCR in our collected normal and MDS patient BMMC samples, and the results showed that their expression levels were consistent with the results of the analysis of MDS patients from the TCGA dataset (Fig. 7D-F), and the expression levels of these molecules were well correlated (Fig. 7G-I). In addition, qRT-PCR revealed that the expression of BC200/miR-150-5p/MYB in human MDS cell lines compared with human normal BMMCs was similar to that in the GEO datasets (Fig. $\mathrm{S} 6 \mathrm{~B}-\mathrm{D})$. Taken together, these results indicated that the dysregulated expression of BC200/miR-150-5p/MYB was a frequent event in MDS and that this positive feedback loop potentially promoted the pathogenesis and development of MDS. 
A

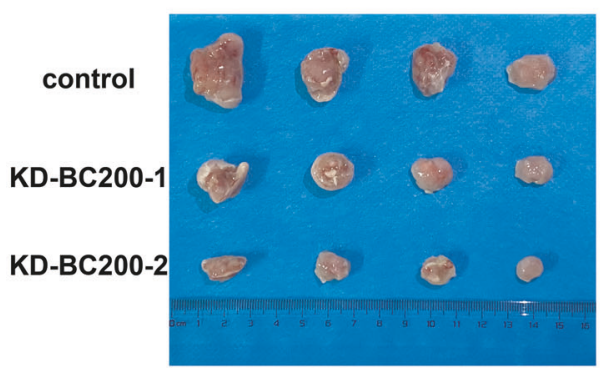

B

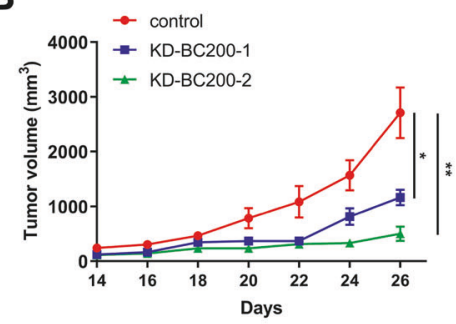

C

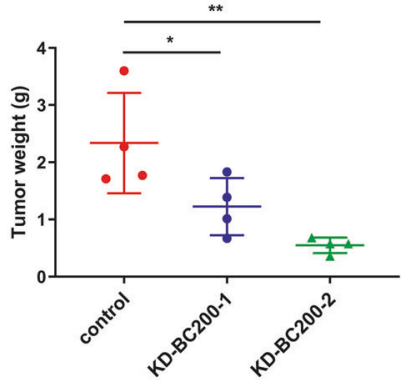

D

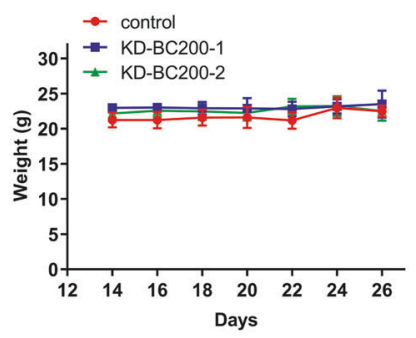

F

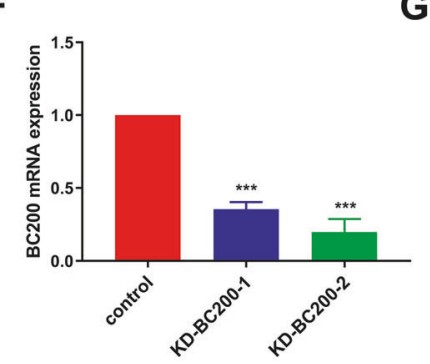

E

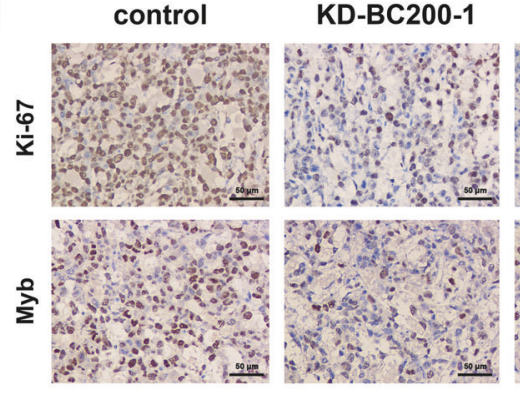

KD-BC200-2

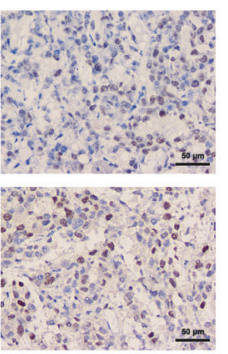

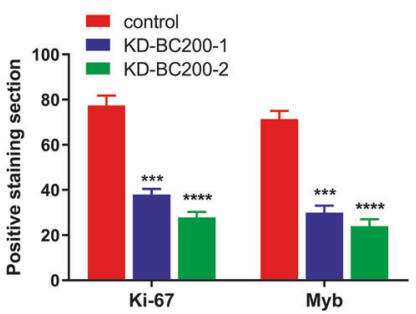

G

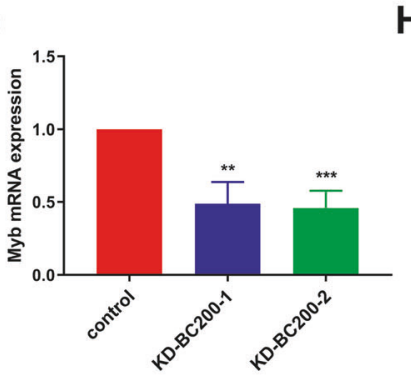

H

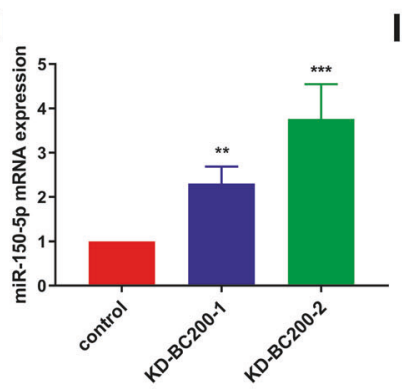

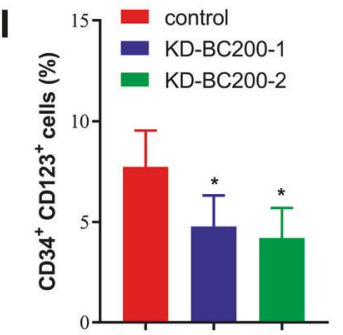

J
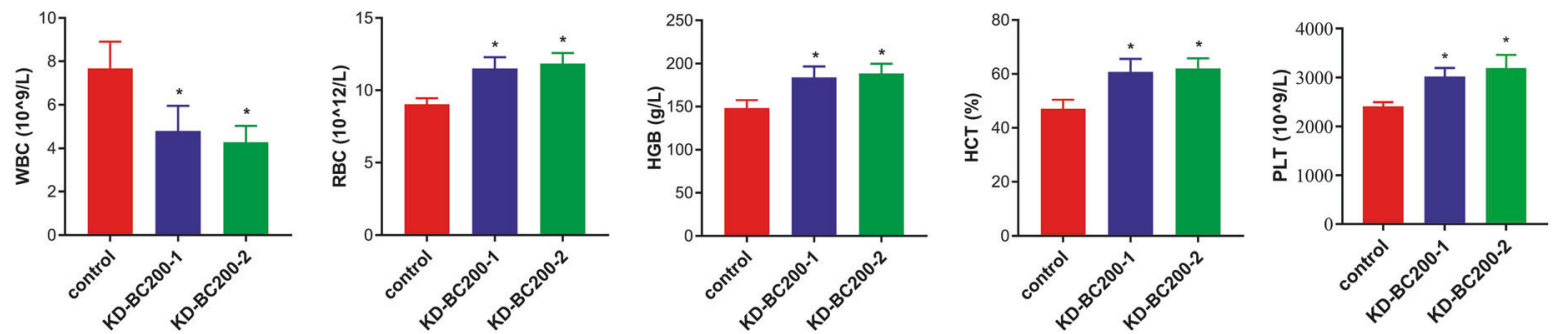

Fig. 6 Knockdown of BC200 inhibits MDS growth in vivo. A SKM-1 cells treated with different BC200 shRNAs $(n=4)$ or control shRNA ( $n=4)$ were inoculated subcutaneously into NCG mice. Tumor growth curves showed that sh-BC200 group led to tumor growth restriction in mice. Scale bar: $1 \mathrm{~cm}$. B Tumor growth curves showed that sh-BC200 group suppressed tumor growth compared with sh-control group. C The subcutaneous tumors were harvested and weighed on the 26th day after implantation. Data are shown as mean \pm SEM. D The NCG mice grew tumors on the fourteenth day after implantation and began to be weighed. E Ki-67 immunostaining of xenograft tissues collected from the BC200-knockdown group and control group. Scale bar: $50 \mu \mathrm{m}$. F-H qRT-PCR was performed on xenograft tissues and was subjected to measure BC200, MYB, and miR-150-5p expression. I Plot of CD123 vs CD34 expression of BM cells of MDS or normal mice being analyzed. J White blood cells (WBCs), red blood cells (RBCs), hemoglobin (HGB), hematocrit (HCT), and platelets (PLTs) counts of MDS or normal mice. ${ }^{*} p<0.05,{ }^{* *} p<0.01,{ }^{* * *} p<0.001,{ }^{* * * *} p<0.0001$.

\section{DISCUSSION}

Several studies have shown that dysregulated IncRNAs play essential roles in the occurrence and development of MDS $[14,27,28]$. In addition to the well-characterized IncRNAs, it is worth exploring potential essential IncRNAs that control the pathogenesis and development of MDS. Thus, we analyzed publicly available databases of DElncRNAs in MDS. Interestingly, we found that carcinogenic BC200 was highly expressed in BMMCs of MDS patients compared with those of normal individuals. Numerous studies have also indicated that BC200 functions as an oncogenic IncRNA, and it has been shown to play important roles in the growth, migration, and invasion of cancer cells. BC200 has become an emerging novel diagnostic marker and therapeutic target in human cancers $[16,29,30]$. Therefore, the abnormal expression and biological function of BC200 in MDS are worth investigating.

Normal cell cycle progression is essential for cell proliferation and differentiation. Disruption or dysregulation of the cell cycle leads to unlimited cell proliferation and inhibition of differentiation and apoptosis, which is closely related to 
A
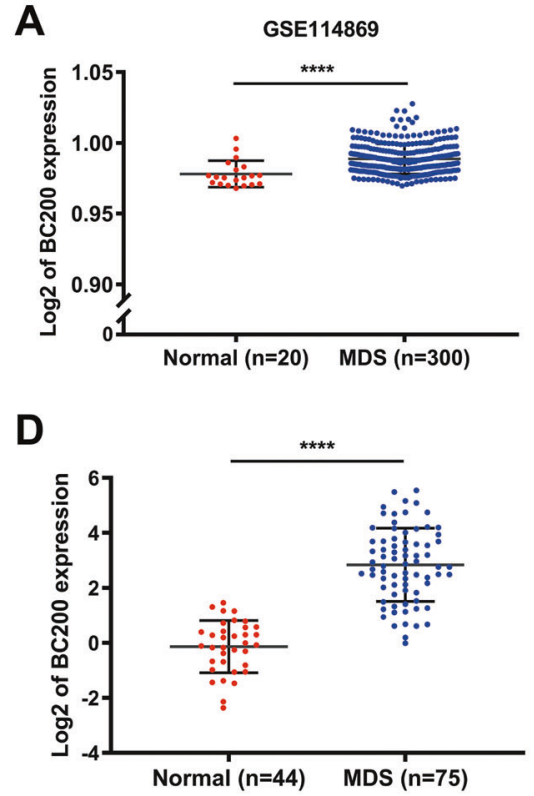

G

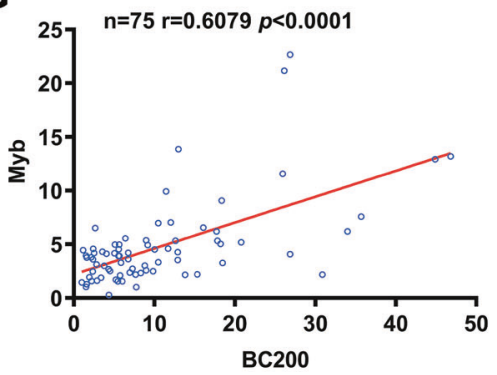

B

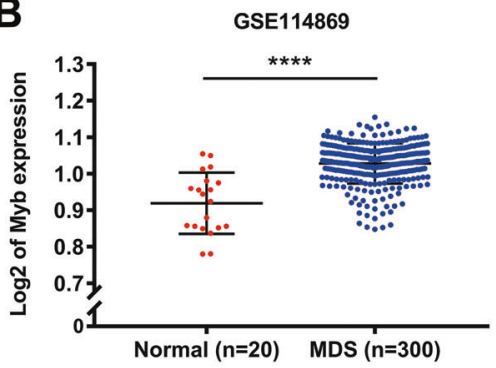

E

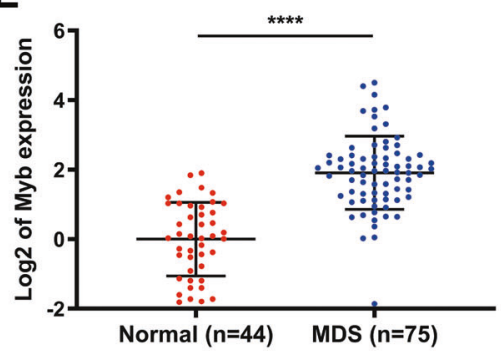

H

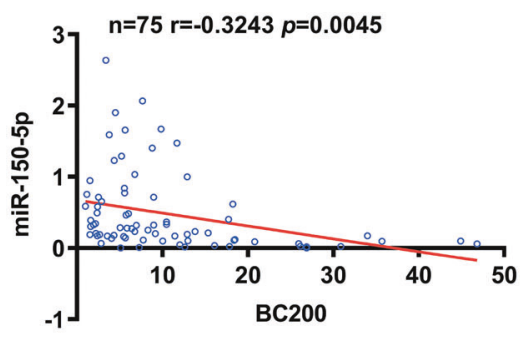

C

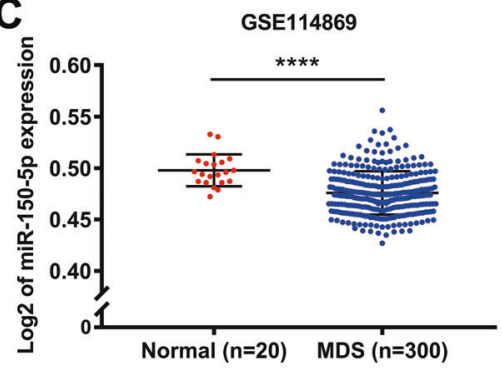

F

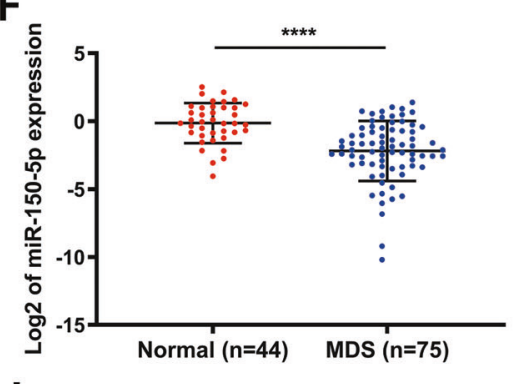

I

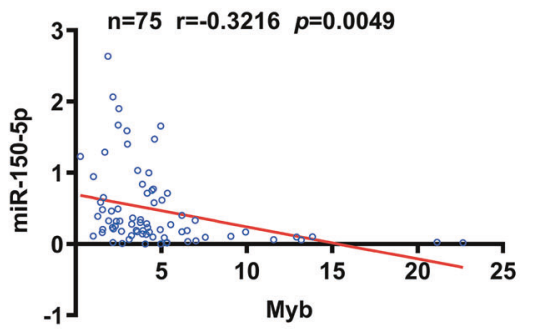

Fig. 7 Clinical significance of the BC200/miR-150-5p/MYB loop based on bioinformatics analysis. A-C Analysis of a GEO dataset (GSE114869) indicated that BC200 and MYB expression was markedly higher in MDS samples than in normal samples. Conversely, miR-150-5p was significantly lower in MDS samples. D-F The expression of BC200, miR-150-5p mRNA, and MYB was analyzed by qRT-PCR in 79 MDS samples and 44 normal samples. G-I Correlation analysis of BC200 and miR-150-5p, MYB and miR-150-5p, and BC200 and MYB expression levels in 75 MDS samples. ${ }^{* * *} p<0.0001$

tumorigenesis. Therefore, we further explored the potential function and mechanism of BC200 in MDS tumorigenesis. Lossof-function assays showed that knockdown of BC200 suppressed proliferation, colony formation, and cell cycle progression but could not induce apoptosis of MDS cells. In vivo experiments demonstrated that BC200 inhibition suppressed the growth and invasiveness of MDS cells in mice. These results indicated that $\mathrm{BC} 200$ plays an important role in the pathogenesis and development of MDS. Moreover, we identified MYB as a novel downstream gene of BC200. Our findings revealed a complex circuitry underlying the concomitant upregulation of BC200 and MYB in MDS cells. Therefore, these findings are of great significance to explore the pathogenesis and treatment of MDS.

In recent years, a new topic of interest in the RNA field has become the ceRNA theory, which proposes a regulatory network between different RNAs, including IncRNAs and miRNAs [31]. As ceRNAs, IncRNAs sponge miRNAs through sequence complementarity and subsequently affect the functional roles of miRNAs [32]. A recent study found that $\mathrm{BC} 200$ could regulate CUEDC2 expression and the PTEN/AKT/p21 pathway by functioning as a ceRNA for sponging miR-619-5p to inhibit glioma progression [33]. In this study, we found that BC200 was mainly located in the cytoplasm. We hypothesized that $B C 200$ regulates MYB by acting as a ceRNA to sponge miRNAs. Bioinformatics analysis and luciferase reporter assays revealed that miR-150-5p is a target of BC200. Moreover,
BC200 silencing markedly upregulated miR-150-5p expression in MDS cells, which confirmed our hypothesis. Previously, miR$150-5 p$ was found to be downregulated in various cancers and has been identified as a tumor suppressor [34, 35]. However, Hussein $\mathrm{K}$ et al. reported that miR-150 was upregulated in MDSdel $(5 q)$, and inhibition of proliferation might contribute to myelodysplastic hematopoiesis via overexpressing miR-150 [36]. The precise functions and underlying molecular mechanisms of miR-150-5p in the context of MDS are still unclear. We demonstrated that overexpression of miR-150-5p in MDS-L and SKM-1 cells could inhibit cell proliferation and induce cell cycle arrest at the $G 1 / G 0$ phase. Our results revealed that the interaction between $\mathrm{BC} 200$ and miR-150-5p plays an important role in MDS and has clinical significance for MDS tumorigenesis, and BC200 exerts its oncogenic effects partly by sponging miR-150-5p in MDS cells.

We further identified the target of miR-150-5p by using bioinformatics analysis and luciferase reporter assays and confirmed MYB as a direct target of miR-150-5p in MDS cells. MYB has been reported to be regulated by miR-150-5p in many disorders [37, 38]. As an essential hematopoietic regulatory transcription factor, MYB is crucial for the proliferation and differentiation of hematopoietic stem cells [39-41]. Dysregulation of MYB activity is often associated with various hematological disorders [42, 43]. Notably, in a zebrafish model, hyperactivity of MYB displayed MDS phenotypes similar to those in human patients [41]. 


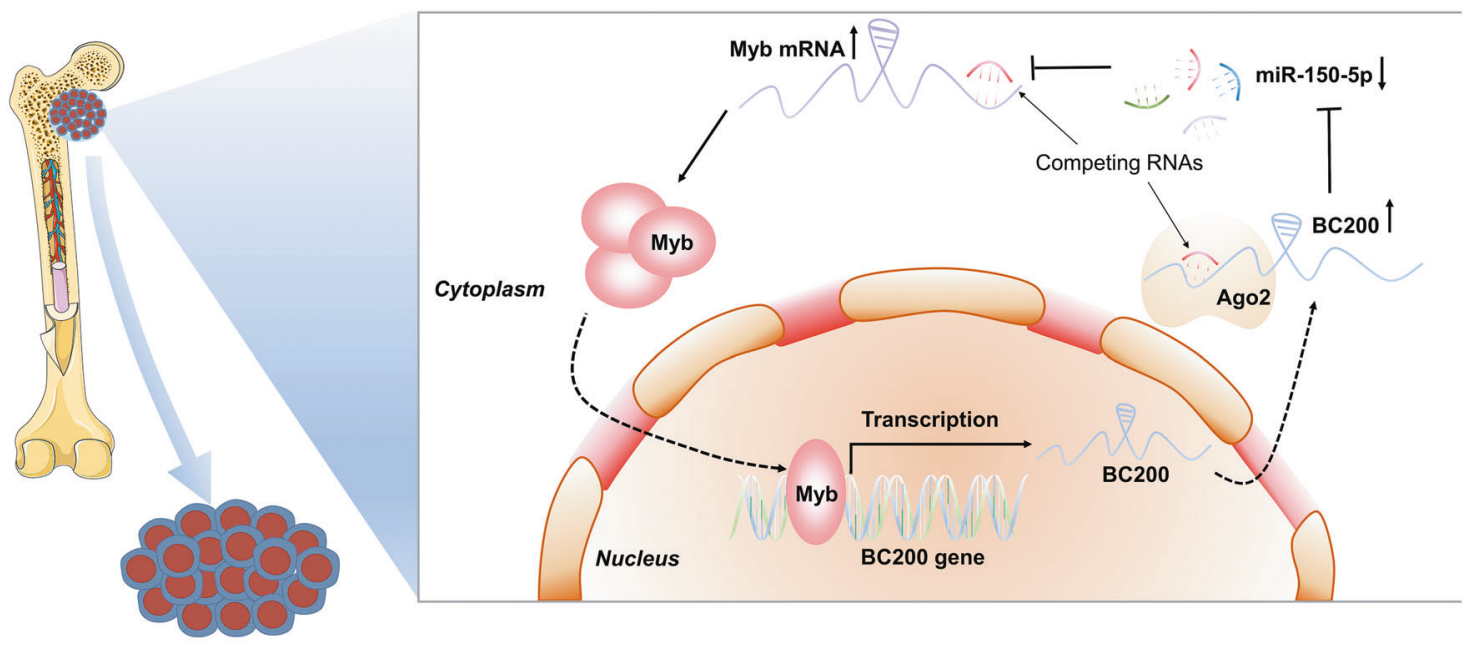

Malignant proliferation

Fig. 8 Schematic diagram of the BC200/miR-150-5p/MYB positive feedback loop in promoting the proliferation of MDS cells. BC200 acts as a miRNA sponge and positively regulates the expression of MYB through sponging miR-150-5p. Reciprocally, MYB can transcriptionally regulate the expression of $\mathrm{BC} 200$, and the overexpression of MYB enhanced the oncogenic effect of $\mathrm{BC} 200 / \mathrm{miR}-150-5 \mathrm{p}$. Therefore, $\mathrm{BC} 200 / \mathrm{miR}-$ $150-5$ p/MYB positive feedback loop is involved in promoting the malignant proliferation of MDS cells.

In this study, we found that overexpression of BC200 in MDS sponged miR-150-5p and further resulted in an increase in MYB mRNA levels. Furthermore, we revealed that MYB was increased in MDS cells and that knockdown of MYB significantly inhibited the proliferation of MDS cells and diminished the proliferationpromoting effect of the miR-150-5p inhibitor in these cells. Therefore, we confirmed that MYB was the functional target of BC200/miR-150-5p in MDS.

Finally, we explored the molecular mechanism by which BC200 is upregulated in MDS. Previous studies have reported that the transcription factor MYB transcriptionally regulates its target genes to act as the critical driver in many biological processes, such as cell proliferation, metastasis, and differentiation [44, 45]. MYB upregulated miR-130a expression and activated the STAT3 and AKT pathways by downregulating NDRG2, which promotes proliferation and metastasis in salivary adenoid cystic carcinoma (SACC) [44]. Moreover, c-MYB could enhance invasion and metastasis through the wnt/ $\beta$-catenin/ axin2 pathway in breast cancer [46]. In acute myeloid leukemia (AML) with inv $(3) / t(3 ; 3)$, it was also found that targeting MYB or mutating its DNA-binding motif within the GATA2 enhancer resulted in myeloid differentiation and cell death, suggesting that interference with transcription of MYB downstream gene EVI1 provides a potential entry point for AML therapy [47]. In addition to a large number of protein-coding genes, many noncoding RNAs, including IncRNAs and miRNAs, are downstream targets of MYB. Lan T et al. reported that MYB enhanced IncRNA SNHG10 expression by regulating SNHG10 promoter activity, which affected SNHG10-driven HCC cell proliferation, invasion, and migration [24]. Herein, we showed that BC200 was a novel transcriptional target of MYB, and ChIP and dualluciferase reporter assays demonstrated that MYB directly transcriptionally regulated $\mathrm{BC} 200$. Overexpression of MYB positively regulated $\mathrm{BC} 200$ abundance and further participated in the malignant growth of MDS cells. Importantly, MYB reversed the oncogenic effect of BC200 on MDS cells. Clinically, we demonstrated that the expression levels of both BC200 and MYB were dramatically higher, while miR-150-5p was significantly lower in MDS samples than in normal samples based on published GEO datasets and our collected patient samples, and the expression levels of these molecules were well correlated.

In conclusion, our study revealed that BC200 is a novel MYBactivated IncRNA. BC200 was highly expressed and played an oncogenic role in MDS cells and mouse models. Mechanistically, BC200 sponged miR-150-5p to attenuate its repressive effect on MYB and promoted the malignant proliferation of MDS cells. This study provided new insight into the function and molecular mechanism of the BC200/miR-150-5p/MYB positive feedback loop in MDS (Fig. 8), indicating that disrupting the BC200/miR-150-5p/MYB loop may be a novel therapeutic approach for MDS.

\section{DATA AVAILABILITY}

The datasets used and/or analyzed during the current study are available from the corresponding author on reasonable request.

\section{REFERENCES}

1. Steensma DP. Myelodysplastic syndromes: diagnosis and treatment. Mayo Clin Proc. 2015;90:969-83.

2. Kennedy JA, Ebert BL. Clinical implications of genetic mutations in myelodysplastic syndrome. J Clin Oncol. 2017;35:968-74.

3. Elnahass $Y$, Youssif L. Cytogenetic features in primary myelodysplastic syndrome Egyptian patients. J Adv Res. 2018;10:77-83.

4. He Q, Zheng Q, Xu F, Shi W, Guo J, Zhang Z, et al. IGF-IR promotes clonal cell proliferation in myelodysplastic syndromes via inhibition of the MAPK pathway. Oncol Rep. 2020;44:1094-104.

5. Kam AYF, Piryani SO, McCall CM, Park HS, Rizzieri DA, Doan PL. Targeting high mobility group box-1 (HMGB1) promotes cell death in myelodysplastic syndrome. Clin Cancer Res. 2019;25:4155-67.

6. Bhan A, Soleimani M, Mandal SS. Long noncoding RNA and cancer: a new paradigm. Cancer Res. 2017;77:3965-81.

7. Wei L, Sun J, Zhang N, Zheng Y, Wang X, Lv L, et al. Noncoding RNAs in gastric cancer: implications for drug resistance. Mol Cancer. 2020;19:62.

8. Cabianca DS, Casa V, Bodega B, Xynos A, Ginelli E, Tanaka Y, et al. A long ncRNA links copy number variation to a polycomb/trithorax epigenetic switch in FSHD muscular dystrophy. Cell 2012;149:819-31.

9. Wang KC, Chang HY. Molecular mechanisms of long noncoding RNAs. Mol Cell. 2011;43:904-14.

10. Pan J, Fang S, Tian H, Zhou C, Zhao X, Tian H, et al. IncRNA JPX/miR-33a-5p/Twist1 axis regulates tumorigenesis and metastasis of lung cancer by activating Wnt/ $\beta$-catenin signaling. Mol Cancer. 2020;19:9.

11. Zhou $M$, Zhao $H$, Xu W, Bao $S$, Cheng $L$, Sun J. Discovery and validation of immune-associated long non-coding RNA biomarkers associated with clinically molecular subtype and prognosis in diffuse large B cell lymphoma. Mol Cancer. 2017;16:16.

12. Prensner JR, Chinnaiyan AM. The emergence of IncRNAs in cancer biology. Cancer Disco. 2011;1:391-407. 
13. Huang $\mathrm{H}-\mathrm{H}$, Chen $\mathrm{F}-\mathrm{Y}$, Chou W-C, Hou H-A, Ko B-S, Lin C-T, et al. Long non-coding RNA HOXB-AS3 promotes myeloid cell proliferation and its higher expression is an adverse prognostic marker in patients with acute myeloid leukemia and myelodysplastic syndrome. BMC Cancer. 2019;19:617.

14. Szikszai K, Krejcik Z, Klema J, Loudova N, Hrustincova A, Belickova M, et al. LncRNA profiling reveals that the deregulation of H19, WT1-AS, TCL6, and LEF1AS1 is associated with higher-risk myelodysplastic syndrome. Cancers (Basel). 2020;12:2726.

15. Shin H, Kim Y, Kim M, Lee Y. BC200 RNA: an emerging therapeutic target and diagnostic marker for human cancer. Mol Cells. 2018;41:993-9.

16. Booy EP, McRae EK, Koul A, Lin F, McKenna SA. The long non-coding RNA BC200 (BCYRN1) is critical for cancer cell survival and proliferation. Mol Cancer. 2017;16:109.

17. Zhai $\mathrm{H}, \mathrm{Li} \mathrm{Y}$. BCYRN1 is correlated with progression and prognosis in gastric cancer. Biosci Rep. 2019. https://doi.org/10.1042/BSR20190505.

18. Chen W, Böcker W, Brosius J, Tiedge H. Expression of neural BC200 RNA in human tumours. J Pathol. 1997;183:345-51.

19. Wu K, Xu K, Liu K, Huang J, Chen J, Zhang J, et al. Long noncoding RNA BC200 regulates cell growth and invasion in colon cancer. Int J Biochem Cell Biol. 2018;99:219-25.

20. Lin $\mathrm{Y}-\mathrm{H}$, Wu M-H, Huang $\mathrm{Y}-\mathrm{H}$, Yeh $\mathrm{C}-\mathrm{T}$, Chi H-C, Tsai C-Y, et al. Thyroid hormone negatively regulates tumorigenesis through suppression of $\mathrm{BC} 200$. Endocr Relat Cancer. 2018;25:967-79.

21. Hu T, Lu Y-R. BCYRN1, a c-MYC-activated long non-coding RNA, regulates cell metastasis of non-small-cell lung cancer. Cancer Cell Int. 2015;15:36.

22. Kong X, Hu S, Yuan Y, Du Y, Zhu Z, Song Z, et al. Analysis of IncRNA, miRNA and mRNA-associated ceRNA networks and identification of potential drug targets for drug-resistant non-small cell lung cancer. J Cancer. 2020;11:3357-68.

23. Batista PJ, Chang HY. Long noncoding RNAs: cellular address codes in development and disease. Cell 2013;152:1298-307.

24. Lan T, Yuan $\mathrm{K}$, Yan $\mathrm{X}, \mathrm{Xu}$ L, Liao $\mathrm{H}$, Hao $\mathrm{X}$, et al. LncRNA SNHG10 facilitates hepatocarcinogenesis and metastasis by modulating its homolog SCARNA13 via a positive feedback loop. Cancer Res. 2019;79:3220-34.

25. Zhang J, Luo N, Luo Y, Peng Z, Zhang T, Li S. microRNA-150 inhibits human CD133-positive liver cancer stem cells through negative regulation of the transcription factor c-Myb. Int J Oncol. 2012;40:747-56.

26. Wu Z, Gao S, Zhao X, Chen J, Keyvanfar K, Feng X, et al. Long noncoding RNAs of single hematopoietic stem and progenitor cells in healthy and dysplastic human bone marrow. Haematologica. 2019;104:894-906.

27. Zhao X, Yin H, Li N, Zhu Y, Shen W, Qian S, et al. An integrated regulatory network based on comprehensive analysis of mRNA expression, gene methylation and expression of long non-coding RNAs (IncRNAs) in myelodysplastic syndromes. Front Oncol. 2019;9:200

28. Li N, Ma Y, Wang W, Yin CC, Wu W, Sun R, et al. LOC101928834, a novel IncRNA in Wnt/ $\beta$-catenin signaling pathway, promotes cell proliferation and predicts poor clinical outcome in myelodysplastic syndromes. Clin Sci (Lond). 2020;134:1279-93.

29. Tan N, Zhu B, Shu H, Tao Y-F, Wu J-R, Fang M, et al. Effect of IncRNA-BC200 on proliferation and migration of liver cancer cells in vitro and in vivo. Oncol Rep. 2020;43:461-70.

30. Ming X-L, Feng Y-L, He D-D, Luo C-L, Rong J-L, Zhang W-W, et al. Role of in hepatocellular carcinoma pathogenesis by IncRNA-miRNA-mRNA network analysis and its diagnostic and prognostic value. Epigenomics. 2019;11:1209-31.

31. Abdollahzadeh R, Daraei A, Mansoori Y, Sepahvand M, Amoli MM, TavakkolyBazzaz J. Competing endogenous RNA (ceRNA) cross talk and language in ceRNA regulatory networks: a new look at hallmarks of breast cancer. J Cell Physiol. 2019;234:10080-100.

32. Thomson DW, Dinger ME. Endogenous microRNA sponges: evidence and controversy. Nat Rev Genet. 2016;17:272-83.

33. Mu M, Niu W, Zhang $X$, Hu S, Niu C. LncRNA BCYRN1 inhibits glioma tumorigenesis by competitively binding with miR-619-5p to regulate CUEDC2 expression and the PTEN/AKT/p21 pathway. Oncogene. 2020;39:6879-92.

34. Fang ZH, Wang SL, Zhao JT, Lin ZJ, Chen LY, Su R, et al. miR-150 exerts antileukemia activity in vitro and in vivo through regulating genes in multiple pathways. Cell Death Dis. 2016;7:e2371.

35. Dai F-Q, Li C-R, Fan X-Q, Tan L, Wang R-T, Jin H. miR-150-5p inhibits non-small-cell lung cancer metastasis and recurrence by targeting HMGA2 and $\beta$-catenin signaling. Mol Ther Nucleic Acids. 2019;16:675-85.

36. Hussein K, Theophile K, Büsche G, Schlegelberger B, Göhring G, Kreipe $H$, et al. Significant inverse correlation of microRNA-150/MYB and microRNA-222/p27 in myelodysplastic syndrome. Leuk Res. 2010;34:328-34.

37. Feng J, Yang $Y$, Zhang $P$, Wang $F$, Ma Y, Qin $H$, et al. miR-150 functions as a tumour suppressor in human colorectal cancer by targeting c-Myb. J Cell Mol Med. 2014;18:2125-34.
38. Cron MA, Maillard S, Truffault F, Gualeni AV, Gloghini A, Fadel E, et al. Causes and consequences of miR-150-5p dysregulation in myasthenia gravis. Front Immunol. 2019;10:539.

39. Pattabiraman DR, Gonda TJ. Role and potential for therapeutic targeting of MYB in leukemia. Leukemia. 2013;27:269-77.

40. Uttarkar S, Dassé E, Coulibaly A, Steinmann S, Jakobs A, Schomburg C, et al. Targeting acute myeloid leukemia with a small molecule inhibitor of the $\mathrm{Myb} /$ p300 interaction. Blood. 2016;127:1173-82.

41. Liu W, Wu M, Huang Z, Lian J, Chen J, Wang T, et al. c-myb hyperactivity leads to myeloid and lymphoid malignancies in zebrafish. Leukemia. 2017;31:222-33.

42. Walf-Vorderwülbecke $V$, Pearce $K$, Brooks $T$, Hubank $M$, van den Heuvel-Eibrink MM, Zwaan CM, et al. Targeting acute myeloid leukemia by drug-induced c-MYB degradation. Leukemia. 2018;32:882-9.

43. Armenteros-Monterroso E, Zhao L, Gasparoli L, Brooks T, Pearce K, Mansour MR, et al. The AAA+ATPase RUVBL2 is essential for the oncogenic function of c-MYB in acute myeloid leukemia. Leukemia. 2019;33:2817-29.

44. Wang $Y$, Zhang $C-Y$, Xia R-H, Han J, Sun B, Sun S-Y, et al. The MYB/miR-130a/ NDRG2 axis modulates tumor proliferation and metastatic potential in salivary adenoid cystic carcinoma. Cell Death Dis. 2018;9:917.

45. Ramaswamy K, Forbes L, Minuesa G, Gindin T, Brown F, Kharas MG, et al. Peptidomimetic blockade of MYB in acute myeloid leukemia. Nat Commun 2018;9:110.

46. Li Y, Jin K, van Pelt GW, van Dam H, Yu X, Mesker WE, et al. c-Myb enhances breast cancer invasion and metastasis through the Wnt/ $\beta$-catenin/Axin2 pathway. Cancer Res. 2016;76:3364-75.

47. Smeenk L, Ottema $S$, Mulet-Lazaro R, Ebert A, Havermans M, Varea AA, et al. Selective requirement of MYB for oncogenic hyperactivation of a translocated enhancer in leukemia. Cancer Disco. 2021;11:2868-83.

\section{AUTHOR CONTRIBUTIONS}

Z.L., P.W., and S.Y. performed and analyzed experiments; J.Z., J.L. and X.X. contributed to the conception and design, financial support, administrative support, and provision of materials; Z.L. and S.Y. analyzed and interpreted the data; Y.W., H.L., L.Z., L.L., Z.W., and B.H. provided technical support; J.L., P.C., and F.W. contributed to the study materials of clinical specimens. Z.L., S.Y., F.Z., J.Z., and J.L. wrote and revised the manuscript. All authors read and approved the final manuscript.

\section{FUNDING}

This work was funded by the National Natural Science Foundation of China (No. $82170138,81870105,81770107,81920108004$, and 82003286), Natural Science Foundation of Hunan Province (No. 2021JJ30022). Scientific Research Fund Project of Hunan Provincial Health Commission (No.20201921), National Key Research and Development Program of China (No.2018YFA0107800), Scientific Research and Innovation Project of postgraduates in Hunan Province (No.CX20200970). The fellowship of China Postdoctoral Science Foundation (No.2020M672474), and the Changsha Municipal Natural Science Foundation (No.kq2014041).

\section{COMPETING INTERESTS}

The authors declare no competing interests.

\section{ETHICS STATEMENT}

The experimental procedures involving clinical samples were obtained under the approval of the Ethics Review Committee of the First Affiliated Hospital of University of South China and Xiangya Hospital of Central South University, and strictly conformed to the Declaration of Helsinki.

\section{ADDITIONAL INFORMATION}

Supplementary information The online version contains supplementary material available at https://doi.org/10.1038/s41419-022-04578-2.

Correspondence and requests for materials should be addressed to Jing Liu, Xiaojuan Xiao or Ji Zhang.

Reprints and permission information is available at http://www.nature.com/ reprints

Publisher's note Springer Nature remains neutral with regard to jurisdictional claims in published maps and institutional affiliations. 
(c) Open Access This article is licensed under a Creative Commons

Attribution 4.0 International License, which permits use, sharing, adaptation, distribution and reproduction in any medium or format, as long as you give appropriate credit to the original author(s) and the source, provide a link to the Creative Commons license, and indicate if changes were made. The images or other third party material in this article are included in the article's Creative Commons license, unless indicated otherwise in a credit line to the material. If material is not included in the article's Creative Commons license and your intended use is not permitted by statutory regulation or exceeds the permitted use, you will need to obtain permission directly from the copyright holder. To view a copy of this license, visit http://creativecommons. org/licenses/by/4.0/.

(c) The Author(s) 2022 